\title{
Predictability of COVID-19 Hospitalizations, Intensive Care Unit Admissions, and Respiratory Assistance in Portugal: Longitudinal Cohort Study
}

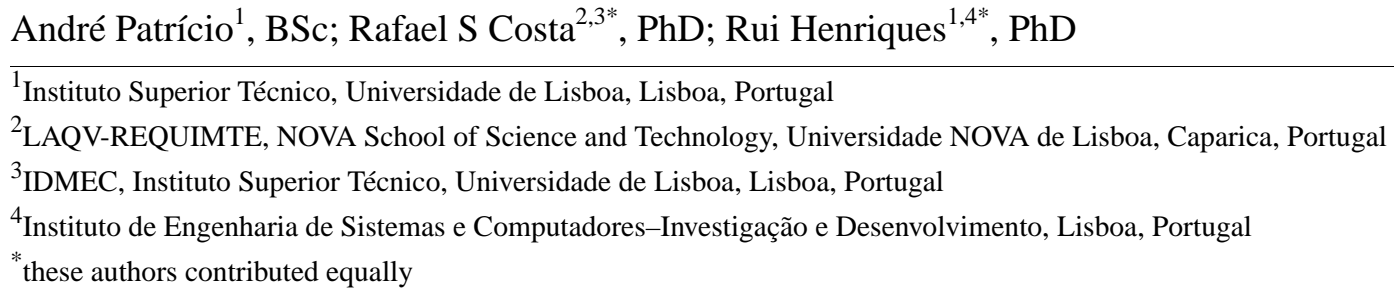

Corresponding Author:

Rafael S Costa, PhD

LAQV-REQUIMTE

NOVA School of Science and Technology

Universidade NOVA de Lisboa

Campus Caparica, 2829-516

Caparica, 2829-516

Portugal

Phone: 351212948351

Email: rs.costa@fct.unl.pt

\section{Abstract}

Background: In the face of the current COVID-19 pandemic, the timely prediction of upcoming medical needs for infected individuals enables better and quicker care provision when necessary and management decisions within health care systems.

Objective: This work aims to predict the medical needs (hospitalizations, intensive care unit admissions, and respiratory assistance) and survivability of individuals testing positive for SARS-CoV-2 infection in Portugal.

Methods: A retrospective cohort of 38,545 infected individuals during 2020 was used. Predictions of medical needs were performed using state-of-the-art machine learning approaches at various stages of a patient's cycle, namely, at testing (prehospitalization), at posthospitalization, and during postintensive care. A thorough optimization of state-of-the-art predictors was undertaken to assess the ability to anticipate medical needs and infection outcomes using demographic and comorbidity variables, as well as dates associated with symptom onset, testing, and hospitalization.

Results: For the target cohort, $75 \%$ of hospitalization needs could be identified at the time of testing for SARS-CoV-2 infection. Over $60 \%$ of respiratory needs could be identified at the time of hospitalization. Both predictions had $>50 \%$ precision.

Conclusions: The conducted study pinpoints the relevance of the proposed predictive models as good candidates to support medical decisions in the Portuguese population, including both monitoring and in-hospital care decisions. A clinical decision support system is further provided to this end.

(J Med Internet Res 2021;23(4):e26075) doi: $\underline{10.2196 / 26075}$

\section{KEYWORDS}

COVID-19; machine learning; intensive care admissions; respiratory assistance; predictive models; data modeling; clinical informatics 


\section{Introduction}

\section{Background}

COVID-19 is a disease caused by the novel coronavirus SARS-CoV-2, transmissible from person to person and associated with acute respiratory complications in severe cases $[1,2]$. The main symptoms of patients infected are fever, cough, and fatigue; others are asymptomatic [3]. The COVID-19 pandemic presents a substantial threat to global health and has been directly responsible for many deaths. Since the first outbreak in December 2019 in Wuhan, China, the number of confirmed infected patients worldwide has exceeded 55 million cases, and nearly 1.3 million people have died from COVID-19 [4]. Current literature has shown that infected patients with specific comorbidities or preconditions (eg, hypertension, respiratory problems, diabetes) and of old age are expected to develop a more severe response to the infection and may consequently need longer hospitalizations and intensive care [5-7]. Strict social confinement measures have been implemented to decrease the COVID-19 $\mathrm{R}_{0}$ value (average number of individuals infected by each infected person) and guarantee the optimal use of equipment and beds at normal, continuous, and intensive care units (ICUs). However, although public health responses aim to delay the spread of the infection, several countries such as the United States, Brazil, Italy, and India have faced severe health care crises.

Without effective antiviral drugs and a vaccine, prognostic tools related to COVID-19 are required. Statistical and computational models could assist clinical staff in triaging patients at high risk for respiratory failure to better guide the allocation of medical resources. Recently, several predictive models ranging from statistical and score-based systems to more recent machine learning models have been proposed in response to COVID-19. Guan et al [8] proposed a Cox regression model to infer potential risk factors associated with serious adverse outcomes in patients with COVID-19. Univariate and multivariate logistic regression models have been used to determine risk factors associated with mortality [9]. Scoring systems have been proposed to predict COVID-19 patient mortality but are limited by small sample sizes, with a poor discriminatory ability [10-12]. Other statistical approaches have also been emerging to aid prognostics $[13,14]$. Complementarily, machine learning methods offer the possibility to model more complex data relationships, generally yielding powerful capabilities to predict outcomes of infectious and noninfectious diseases in medical practice [15-17]. To this end, classification and regression models have been proposed for risk stratification of patients and to screen the spread of COVID-19 [18-20]. Despite the inherent potentialities of ongoing efforts, studies in the context of COVID-19 are limited by either the size of available cohorts or the lack of a systematic comparison of different models [21-24], and generally neglect the predictability of medical needs (instead the focus is commonly placed on measurable disease factors, early detection of infection, and mortality risk prediction [25-28]). None of these studies have comprehensively targeted the Portuguese population at the present time.

\section{This Study}

This study provides a structured view on the predictability of hospitalizations, ICU admissions, respiratory assistance needs, and survivability outcomes using a retrospective cohort encompassing individuals with a SARS-CoV-2-positive result in Portugal as of June 30, 2020.

To this end, and considering demographic, comorbidity, and care provision variables collected for the infected individuals, an assessment methodology was conducted, whereby state-of-the-art predictive models were hyperparameterized and robustly evaluated in order to assess the upper bounds on the predictive performance for each one of the targeted variables. In addition, whenever applicable, this analysis was extended toward the various stages of a patient's cycle: prehospitalization (at the time of testing), after hospitalization, and after ICU admission.

This study offers a solid methodology for the robust assessment of the predictability guarantees of future care needs of infected individuals, contrasting with the dominant correlation-based guarantees in literature. As comparable studies demonstrated in other populations, it lays a solid ground to compare type-I and type-II predictive errors and assess population-wise differences.

\section{Methods}

\section{Overview}

Complete subpopulations from the target cohort were identified for each output (Figure 1), guaranteeing the presence of all individuals undertaking the target forms of care (hospitalization, ICU admission, respiratory support) with a recovery-or-death outcome.

After the sampling and data curation steps (Figure 1), we proceeded to the optimization of data preprocessing options and classifiers' parameterization for each of the target variables separately. To this end, we applied a nested 10-fold cross-validation assessment methodology, whereby we first create train-test partitions (outer cross-validation) to assess the performance of an optimized classification method, and within each training fold we further create train-test partitions (inner cross-validation) for hyperparameterizing the predictive model under assessment. This methodology guarantees that all observations are used to assess the final performance and prevents biases as hyperparameterization takes place within each training folds.

Within each inner train-test fold, Bayesian optimization [29] was applied to find the hyperparameters that best fit the pipeline. The optimization measures are:

- $\quad$ F1 score and $0.7 \times$ recall $+0.3 \times$ precision for binary classes. These two views generate two sets of classifiers: one that equally weights recall-and-precision views, and other that, similar to the $\mathrm{F} 2$ score $\left(\mathrm{F}_{\beta}\right.$, where $\left.\beta=2\right)$, prioritizes the optimization of the true-positive rate (recall) at the cost of a lower positive predictive value (precision);

- Cohen kappa and average class recall for target variables with more than 2 classes (respiratory support). 
Figure 1. Exclusion and inclusion criteria for composing the outcome-conditional cohorts: hospitalization, respiratory assistance support, intensive care unit (ICU) admission, and survivability. Blank/unknown cells in the Direcção-Geral da Saúde data set are classified as missing values.

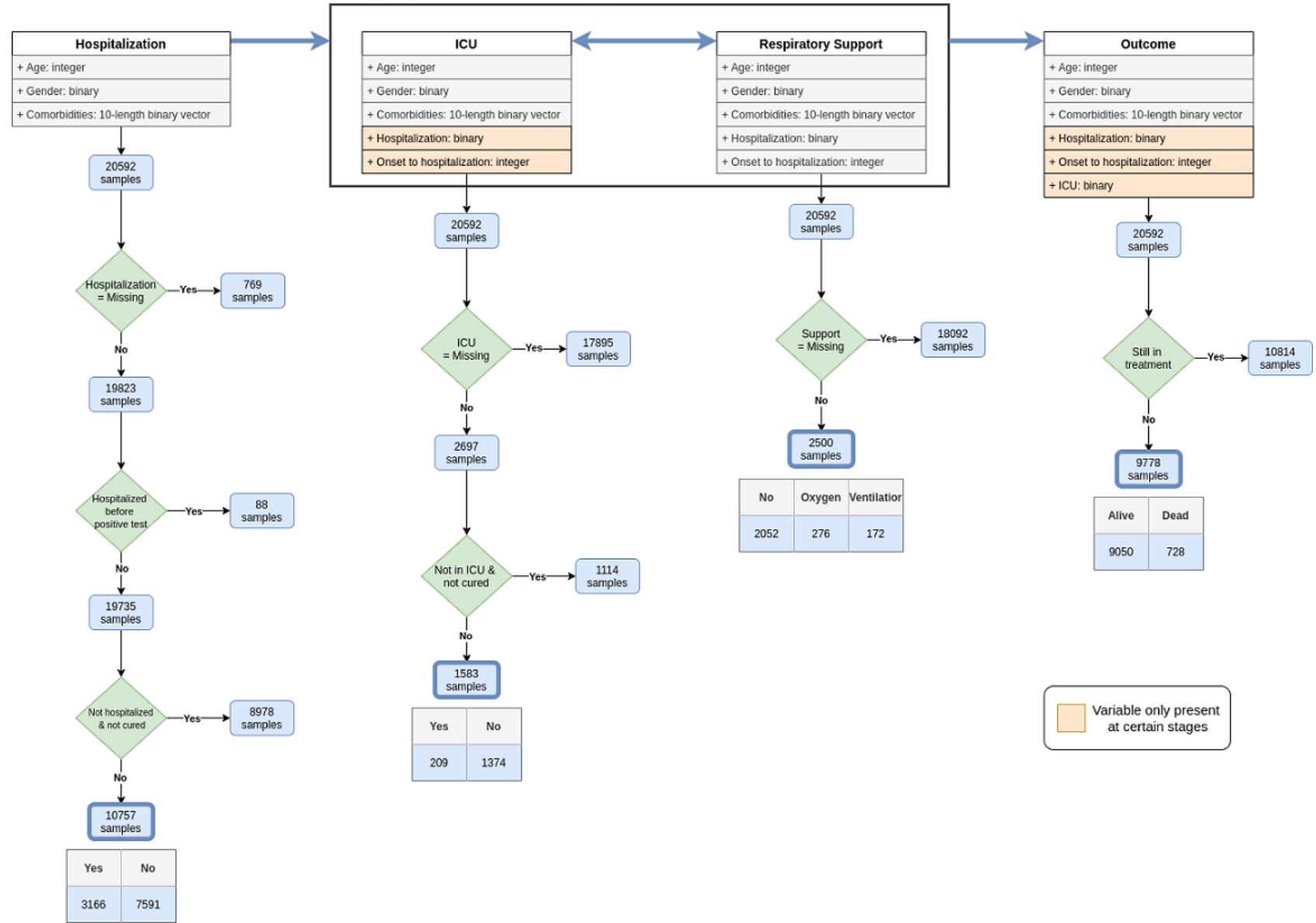

Hospitalization, UCI admission, respiratory support, and recovery-or-death outcomes for SARS-CoV-2-infected individuals are considerably imbalanced, hence, the relevance of the placed recall-precision and multiclass recall views. In particular, considering both a balanced recall-precision optimization and recall-oriented optimization is relevant for clinical decisions. When the allocated teams have capacity to remotely monitor SARS-CoV-2-infected patients, the predictive models optimized with a schema that prioritizes recall should be pursued to guarantee that no vulnerable patient is left out. Nevertheless, when monitoring capacity is limited, greater attention to precision is necessary, and only more vulnerable patients (as suggested by the predictive models optimized with balanced recall-precision views) should be attemptively monitored.

The allowed preprocessing options are as follows: imputation of missing values using median-mode imputation, KNNImputer, or none; class balancing using subsampling, oversampling, SMOTE (Synthetic Minority Oversampling Technique), or none; and normalization of real-valued variables using standardization, scaling, or none. The selected classifiers are as follows: Bernoulli naive Bayes, Gaussian naive Bayes, k-nearest neighbors (KNN), decision tree (DT), random forest, XGBoost (XGB), logistic regression, Light Gradient Boosting Machine (LightGBM), Super Learner, and multilayer perceptron (MLP). Super Learner uses folding to hyperparameterize models and selects predictors for out-of-fold predictions from individual performance estimates per fold. In this context, Super Learner's performance is generally coincident with the best predictive model and thus not always disclosed in the Results section to allow the identification of the best underlying predictors. We considered the implementations provided in the scikit-learn [30] and xgboost [31] packages in Python (Python Software Foundation). For each classifier, all supported parameters in scikit-learn were subjected to hyperparameterization. Regarding the MLP, we placed upper limits on the number of hidden layers (3) and nodes per layer (20) given the low-dimensionality nature of the target data set. The hyperparameters were subjected to a total of 50 iterations. Multimedia Appendix 1 displays the optimized parameters for the best-performing predictive models per outcome.

Differences in performance from the paired-error estimates collected per fold were statistically tested using $t$ tests when estimates passed the Shapiro-Wilk normality test. When this condition was not satisfied, Wilcoxon signed-rank tests were applied.

In addition to the conducted analysis, the best predictors trained on the whole data set were made available within a clinical decision support system built using flask technology and dash facilities in Python [32], which can run as an offline web application.

\section{Data Source}

A retrospective cohort (from March 1 to June 30, 2020) of patients with confirmed COVID-19 in Portugal was used in this 
study. The anonymized data set was provided by the Directorate General of Health (Direcção-Geral da Saúde, DGS), the Portuguese health authority. The gathered data, called the covid19-DGS database, contains information pertaining to the demographic and clinical patient characteristics as well as preexisting conditions.

Data are available upon reasonable request.

\section{Ethical Considerations}

The COVID-19 data set is provided by the DGS under the collaborative score $4 C O V I D$ research project proposal. The tasks conducted in the score 4 COVID project were further validated by the Ethical Committee of the NOVA School of Science and Technology.

\section{Results}

Results on the predictability of hospitalization needs, ICU admissions, respiratory assistance, and outcome of infected individuals living in Portugal, as of June 30, 2020, are discussed below.

\section{Cohort Characteristics}

The target cohort comprised 38,545 individuals who were SARS-CoV-2 positive: 17,046 recoveries (SARS-CoV-2 negative after positive testing) and 1155 deaths. Four individuals were excluded from the data set due to inconsistent recordings related to age and pregnancy-gender variables. Table 1 provides essential statistics. Figure 2 further describes sex and age distributions in deaths, hospitalizations, ICU admissions, and average number of days from symptom onset (traced by the public health line for COVID-19) to a positive test result and hospitalization.

Within the target population, there were 4326 hospitalizations (11.2\% of population base) and 253 admissions to the ICU (5.8\% of hospitalizations). Among ICU internments, there were 82 recoveries and 61 deaths. In terms of respiratory support, a total of 180 individuals undertook assisted ventilation, 292 submitted to oxygen therapy, and 9 underwent alternative modes of respiratory support such as extracorporeal membrane oxygenation.

The major classes of comorbidities monitored were neoplasm, diabetes, asthma, pulmonary, hepatic, hematological, renal, neurological, neuromuscular, and immune deficiency conditions. The representativity of individuals with one or more comorbidities, as well as their impact on survivability, is depicted in Figure 3. 
Table 1. Characteristics of SARS-CoV-2-infected patients in the target cohort.

\begin{tabular}{|c|c|}
\hline Characteristic & Value \\
\hline \multicolumn{2}{|l|}{ Numeric variables, mean (SD); range } \\
\hline Age at notification (years) & $48.3(22.1) ; 0-105$ \\
\hline Onset to hospitalization (days) & $1.1(5.1) ; 0-169$ \\
\hline \multicolumn{2}{|l|}{ Categoric variables, $n(\%)$} \\
\hline \multicolumn{2}{|l|}{ Gender } \\
\hline Female & $11,252(54.64)$ \\
\hline Male & $9340(45.36)$ \\
\hline \multicolumn{2}{|l|}{ Hospitalization } \\
\hline Yes & $16,651(84.00)$ \\
\hline No & $3172(16.00)$ \\
\hline \multicolumn{2}{|l|}{ ICU ${ }^{\mathrm{a}}$ admission } \\
\hline Yes & $209(7.75)$ \\
\hline No & $2488(92.25)$ \\
\hline \multicolumn{2}{|l|}{ Respiratory support } \\
\hline Oxygen therapy & $276(11.04)$ \\
\hline Assisted ventilation & $172(6.88)$ \\
\hline No support & $2052(82.08)$ \\
\hline \multicolumn{2}{|l|}{ Comorbidities } \\
\hline Cancer & $940(4.56)$ \\
\hline Cardiac disease & 3025 (14.69) \\
\hline Diabetes & $2134(10.36)$ \\
\hline Immune deficiency & $222(1.08)$ \\
\hline Renal disease & $718(3.49)$ \\
\hline Liver disease & $206(1.00)$ \\
\hline Lung disease & $794(3.86)$ \\
\hline Chronic neurological disease & $1087(5.28)$ \\
\hline \multicolumn{2}{|l|}{ Mortality } \\
\hline Yes & $728(7.45)$ \\
\hline No & $9050(92.55)$ \\
\hline
\end{tabular}

${ }^{\mathrm{a}} \mathrm{ICU}$ : intensive care unit. 
Figure 2. Cohort statistics: (A-C) demographic distribution of infected individuals with known outcome (death and recovery) and stage in the care life cycle (hospitalization and intensive care unit [ICU] admission); (D) average number of days between care stages (the plotted negative bin [ie, negative occurrences] corresponds to hospitalizations before SARS-CoV-2 testing).

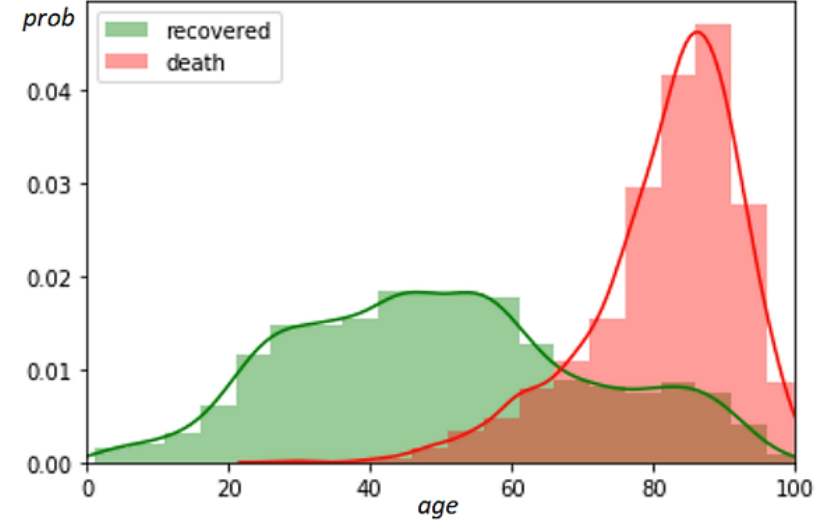

(A) age distribution of recoveries and death

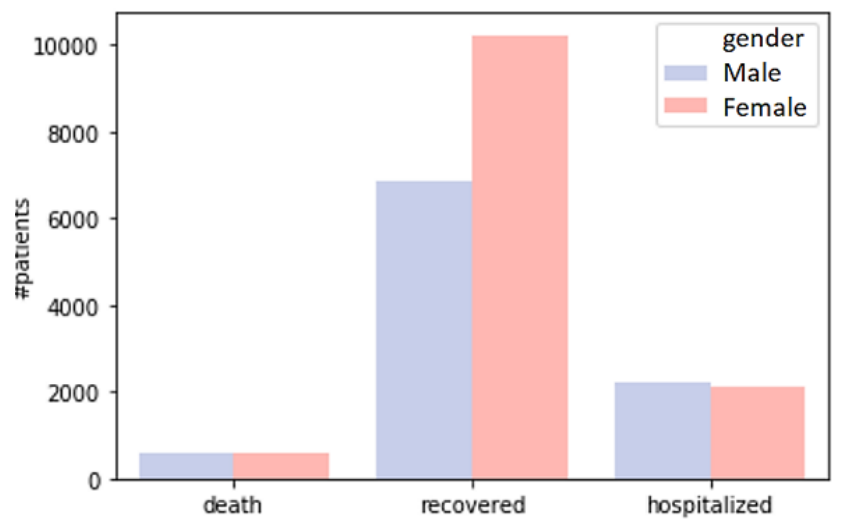

(C) sex distribution

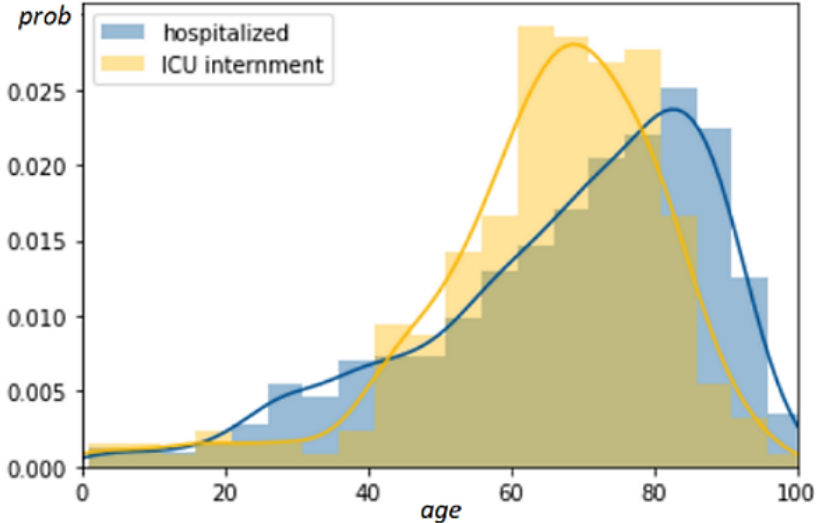

(B) age distribution of hospitalizations and ICU internments

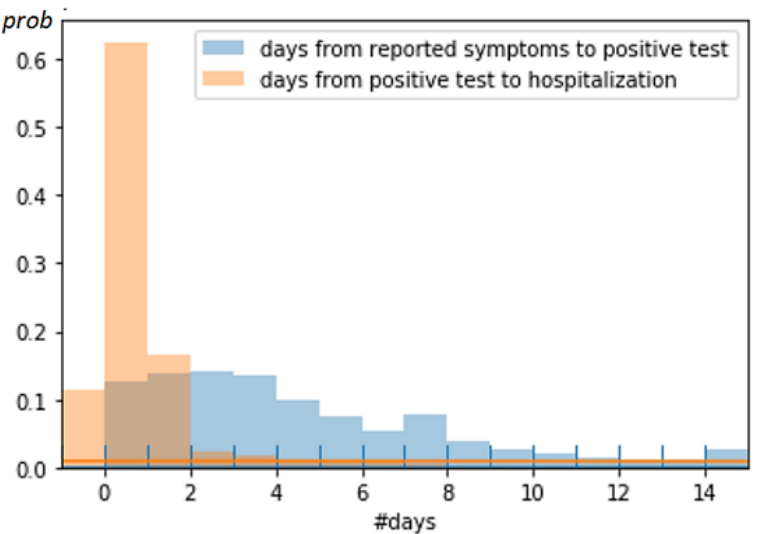

(D) days from symptom to test and from test to hospitalization

Figure 3. Cohort statistics: (A) distribution of individuals with one or more comorbidities among deaths, recovered cases, and hospitalizations; and (B) association between individual comorbidities and survivability outcomes.

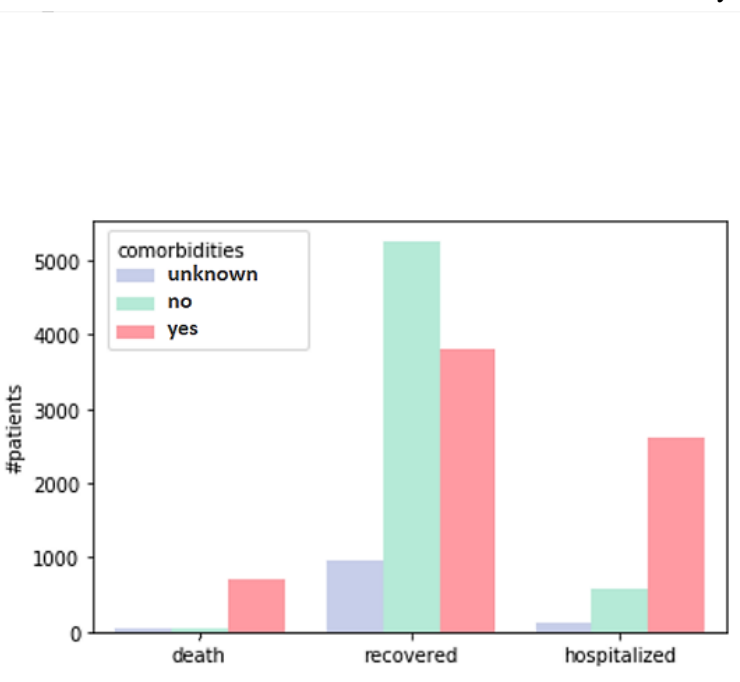

(A) distribution of individuals with comorbidities

\section{Hospitalization}

Figure 4 and Table 2 provide results pertaining to the models' ability to predict the need for individuals to be hospitalized once
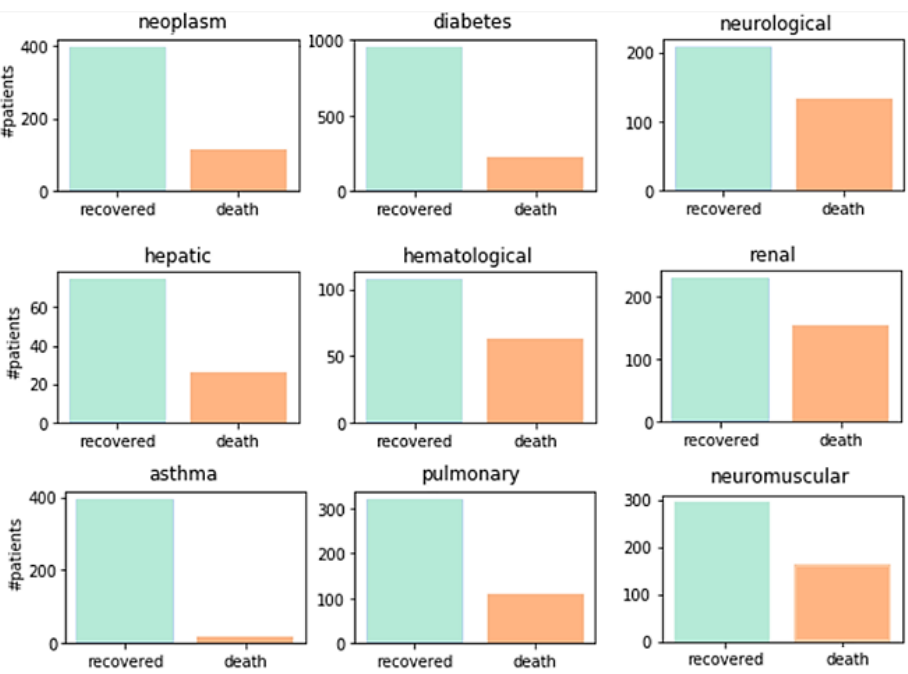

(B) comorbidity impact on survivability

they are tested as SARS-CoV-2 positive given their (1) demographic group (age and gender) and (2) comorbidity factors. Comorbidity factors were categorized in accordance with the presence or absence of kidney, asthma, lung, cancer, 
neuromuscular, diabetes, HIV, cardiac, and pregnancy conditions. Nonhospitalized individuals without a clear outcome (recovery or death) were excluded from this analysis. Figure 5 provides the receiver operating characteristic curve per predictor for each optimization setting.

Generally, we observed that nearly $90 \%$ of hospitalization needs could be identified at the time of SARS-CoV-2 testing. This level of recall/sensitivity was observed at the expense of an approximate $55 \%$ precision, meaning that more than half of the predicted hospitalization needs were in fact observed. Logistic regression and MLP were the best-performing classification models according to F1-score and recall, respectively. Statistical superiority was verified for logistic regression but not MLP against peer models (at $=.05$ ). These results provide empirical evidence toward the role of these predictors in supporting individual remote monitoring decisions.

Figure 4. Predictability of hospitalizations for individuals testing SARS-CoV-2 positive. Recall, precision, and F1 for the best predictors in F1 (left) and recall-oriented (right) scores on the validation set after nested cross-validation. MPL: multilayer perceptron.

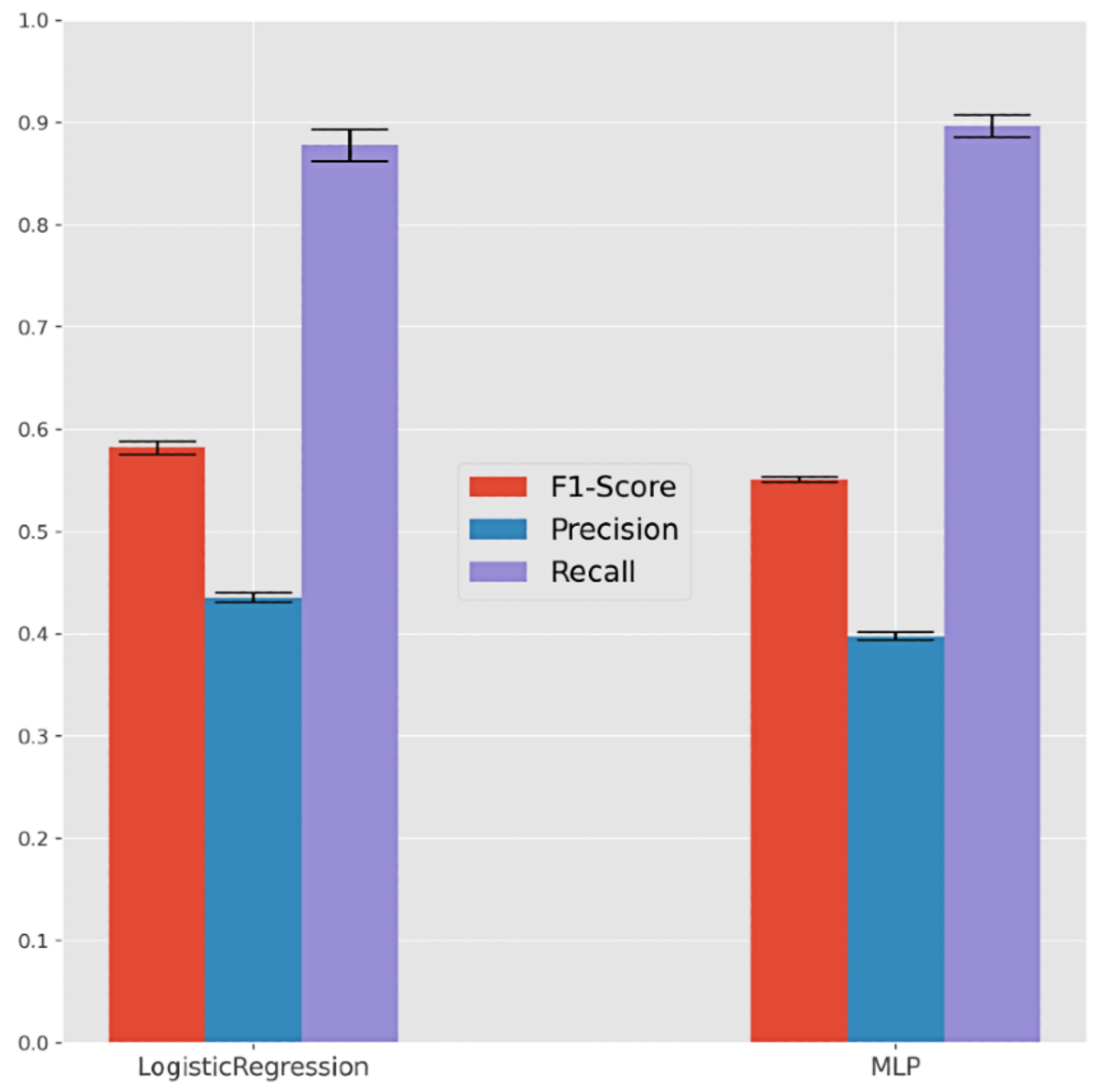


Table 2. Predictability of hospitalizations per predictive model.

\begin{tabular}{|c|c|c|c|c|}
\hline \multirow[t]{2}{*}{ Model } & \multicolumn{2}{|c|}{ F1 optimization, mean (SD) } & \multicolumn{2}{|c|}{ Recall-oriented $\left(\mathrm{F}_{\beta=2}\right)$ optimization, mean (SD) } \\
\hline & F1-score & Recall & F1-score & Recall \\
\hline $\mathrm{KNN}^{\mathrm{a}}$ & $0.544(0.007)$ & $0.883(0.020)$ & $0.545(0.005)$ & $0.890(0.017)$ \\
\hline $\mathrm{DT}^{\mathrm{b}}$ & $0.562(0.030)$ & $0.837(0.069)$ & $0.548(0.004)$ & $0.897(0.007)$ \\
\hline $\mathrm{RF}^{\mathrm{c}}$ & $0.535(0.010)$ & $0.878(0.016)$ & $0.541(0.005)$ & $0.874(0.029)$ \\
\hline $\mathrm{XGB}^{\mathrm{d}}$ & $0.546(0.004)$ & $0.897(0.012)^{\mathrm{e}}$ & $0.545(0.004)$ & $0.895(0.011)$ \\
\hline$L^{f}$ & $0.582(0.006)^{\mathrm{e}}$ & $0.878(0.015)$ & $0.583(0.010)^{\mathrm{e}}$ & $0.879(0.015)$ \\
\hline $\mathrm{MLP}^{\mathrm{g}}$ & $0.549(0.006)$ & $0.892(0.010)$ & $0.551(0.003)$ & $0.897(0.011)^{\mathrm{e}}$ \\
\hline LGBM $^{\mathrm{h}}$ & $0.545(0.005)$ & $0.893(0.013)$ & $0.545(0.005)$ & $0.893(0.016)$ \\
\hline
\end{tabular}

${ }^{\mathrm{a}} \mathrm{KNN}$ : k-nearest neighbors.

${ }^{\mathrm{b}} \mathrm{DT}$ : decision tree.

${ }^{\mathrm{c}} \mathrm{RF}$ : random forest.

${ }^{\mathrm{d}}$ XGB: XGBoost.

${ }^{\mathrm{e}}$ Best-performing models

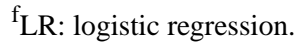

${ }^{\mathrm{g}}$ MLP: multilayer perceptron.

${ }^{\mathrm{h}}$ LGBM: LightGBM.

Figure 5. Receiver operating characteristic curves with the predictive behavior of the selected classifiers in asserting hospitalization needs at the time of SARS-CoV-2 testing. XGB: XGBoost, MLP: multilayer perceptron, LGBM: LightGBM.

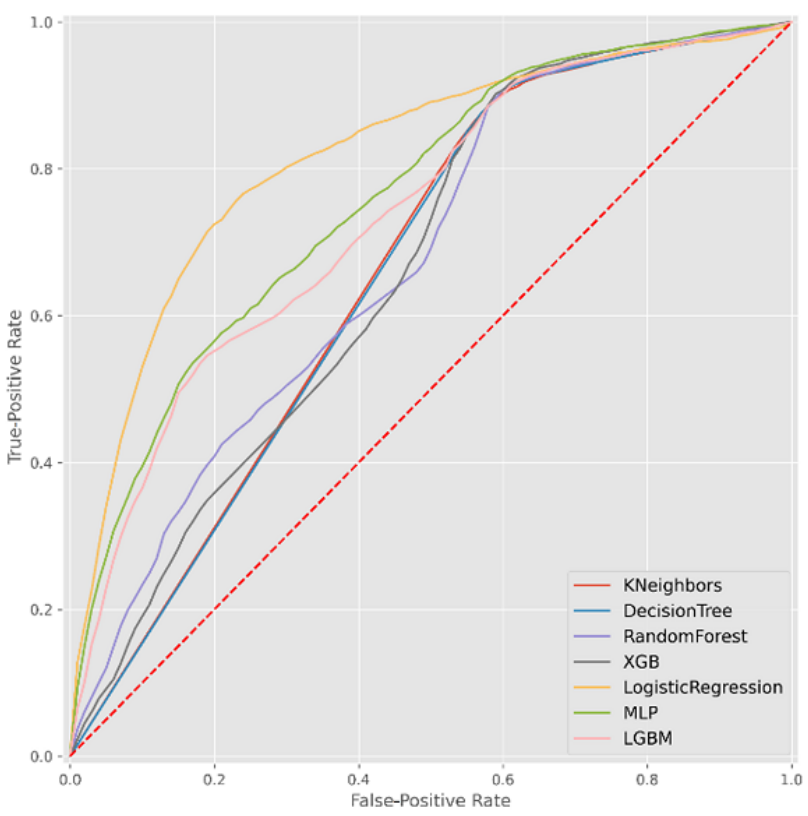

(A) F1 optimization

\section{ICU Admissions}

Figures 6 and 7 and Table 3 assess the ability to anticipate intensive care needs for infected individuals at two stages: before hospitalization and after hospitalization. To this end, the

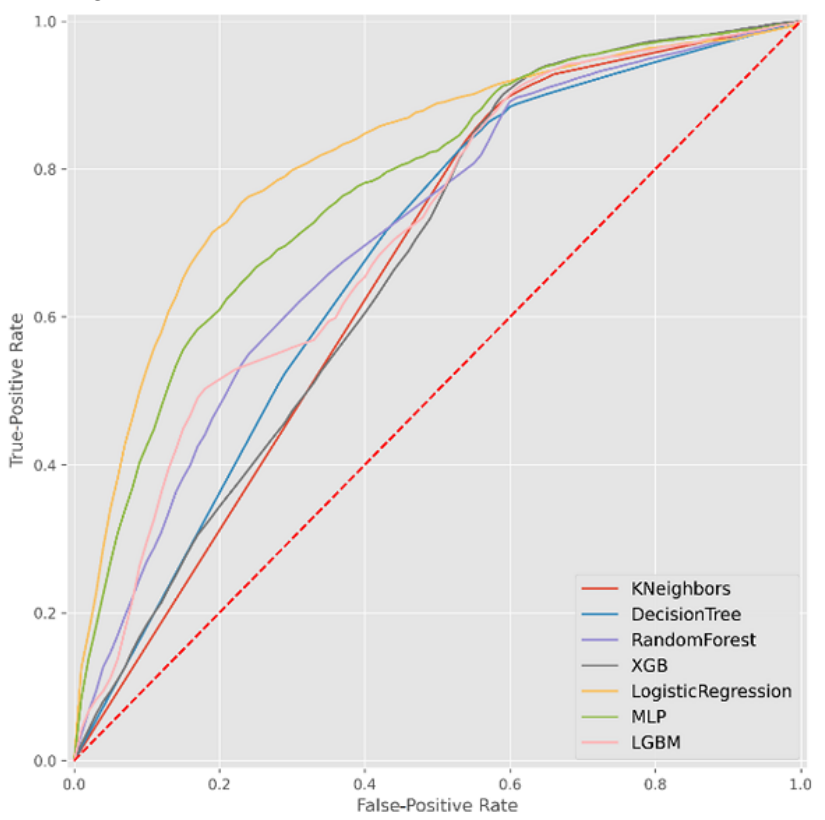

(B) $F \beta=2$ (recall-oriented) optimization

proposed methodology was pursued considering demographic factors, comorbidity factors, and the time to hospitalization for hospitalized individuals. Individuals without a SARS-CoV-2-negative test result after infection were excluded. 
Figure 6. Predictability of intensive care unit admission. Results for the best F1 predictor (left) and recall-oriented predictor (right). XGB: XGBoost.

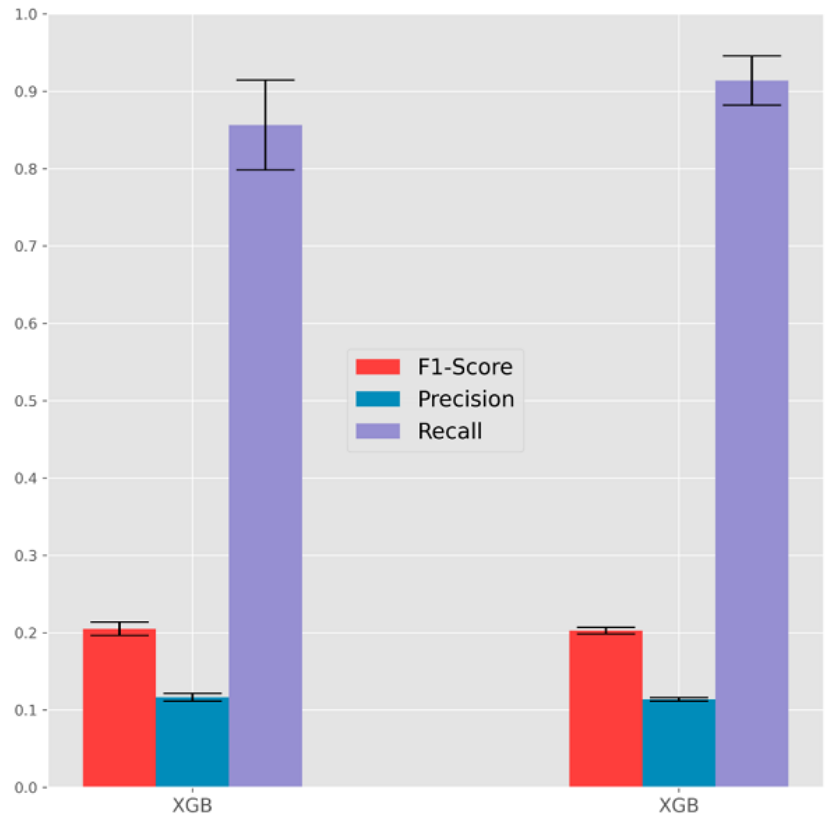

(A) Prehospitalization

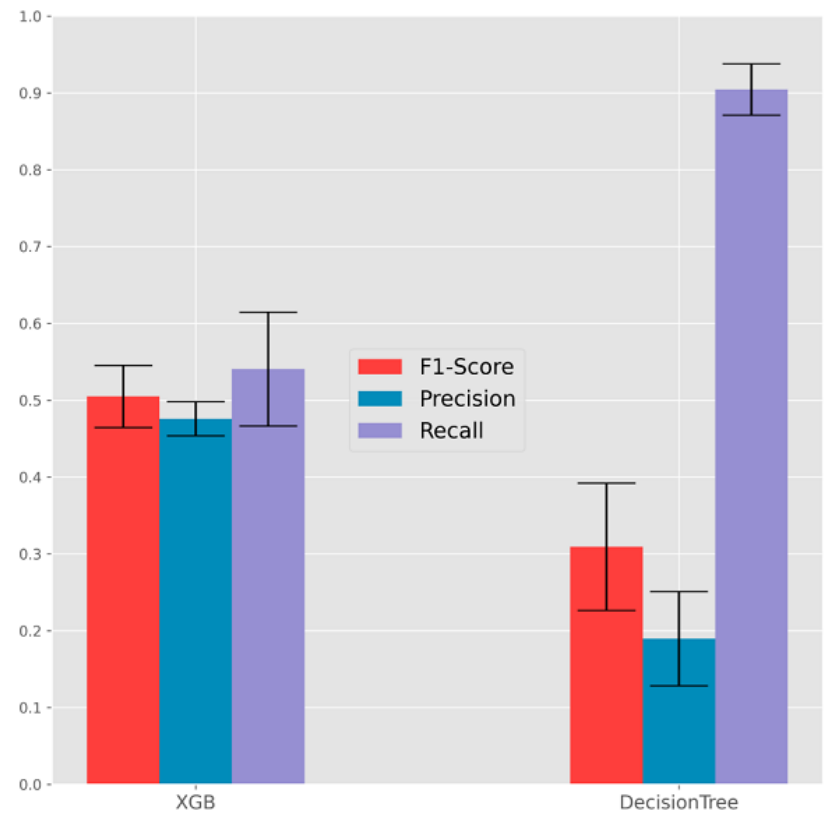

(B) Posthospitalization

Figure 7. Receiver operating characteristic curves with the predictive behavior of the selected classifiers in predicting intensive care unit admission needs at the time of hospitalization. XGB: XGBoost, MLP: multilayer perceptron, LGBM: LightGBM.

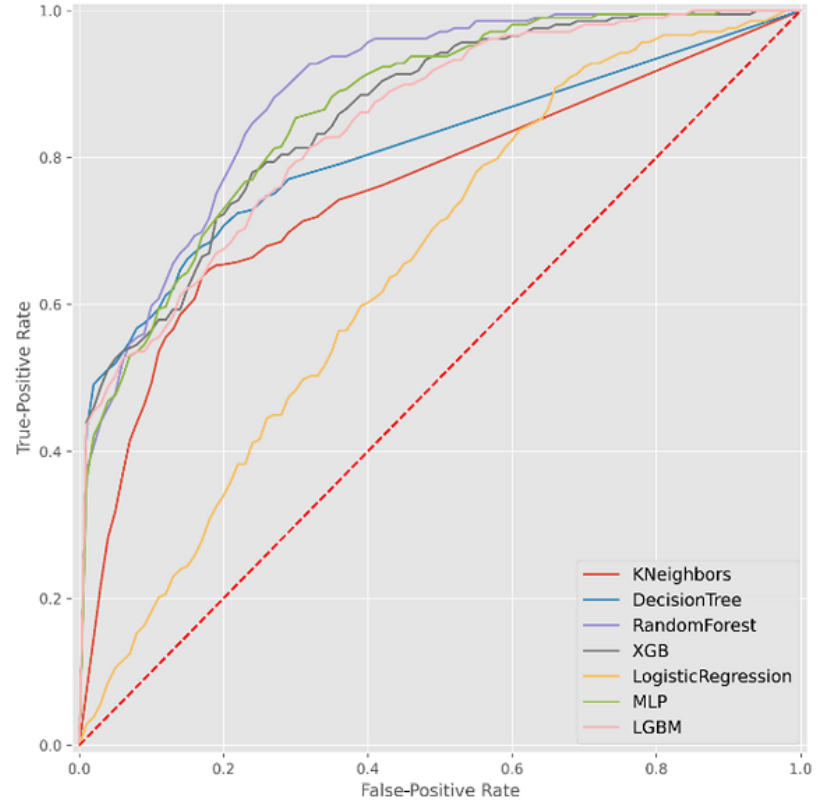

(A) F1 optimization

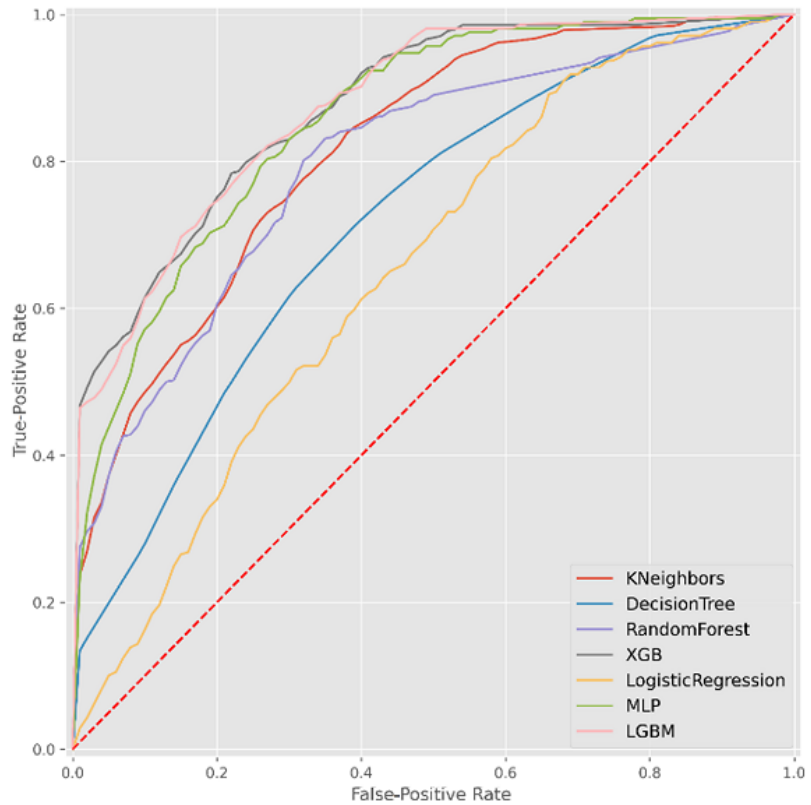

(B) $F \beta=2$ (recall-oriented) optimization 
Table 3. Predictability of intensive care unit admissions per predictive model.

\begin{tabular}{|c|c|c|c|c|}
\hline \multirow[t]{2}{*}{ Model } & \multicolumn{2}{|c|}{ F1 optimization, mean (SD) } & \multicolumn{2}{|c|}{$\mathrm{F}_{\beta=2}$ (recall-oriented) optimization, mean (SD) } \\
\hline & F1-score & Recall & F1-score & Recall \\
\hline \multicolumn{5}{|c|}{ At the time of hospitalization } \\
\hline $\mathrm{KNN}^{\mathrm{a}}$ & $0.428(0.039)$ & $0.574(0.156)$ & $0.369(0.037)$ & $0.741(0.088)$ \\
\hline $\mathrm{DT}^{\mathrm{b}}$ & $0.461(0.016)$ & $0.651(0.056)$ & $0.309(0.083)$ & $0.904(0.033)^{\mathrm{c}}$ \\
\hline $\mathrm{RF}^{\mathrm{d}}$ & $0.454(0.027)$ & $0.713(0.088)^{\mathrm{c}}$ & $0.382(0.103)$ & $0.794(0.128)$ \\
\hline $\mathrm{XGB}^{\mathrm{e}}$ & $0.505(0.040)^{\mathrm{c}}$ & $0.541(0.074)$ & $0.431(0.040)$ & $0.766(0.084)$ \\
\hline$L^{f}$ & $0.250(0.015)$ & $0.622(0.041)$ & $0.248(0.013)$ & $0.651(0.074)$ \\
\hline$M L P^{g}$ & $0.449(0.060)$ & $0.703(0.145)$ & $0.410(0.039)$ & $0.818(0.050)$ \\
\hline LGBM $^{\mathrm{h}}$ & $0.480(0.023)$ & $0.536(0.048)$ & $0.435(0.025)^{\mathrm{c}}$ & $0.770(0.051)$ \\
\hline \multicolumn{5}{|c|}{ At the time of SARS-CoV-2 testing } \\
\hline $\mathrm{KNN}$ & $0.195(0.016)$ & $0.818(0.090)$ & $0.198(0.007)$ & $0.852(0.039)$ \\
\hline DT & $0.209(0.012)$ & $0.752(0.135)$ & $0.201(0.007)$ & $0.890(0.036)$ \\
\hline $\mathrm{RF}$ & $0.200(0.008)$ & $0.880(0.038)$ & $0.200(0.008)$ & $0.880(0.044)$ \\
\hline $\mathrm{XGB}$ & $0.205(0.009)$ & $0.857(0.058)$ & $0.203(0.004)$ & $0.914(0.032)$ \\
\hline LR & $0.200(0.008)$ & $0.847(0.054)$ & $0.201(0.007)$ & $0.880(0.034)$ \\
\hline MLP & $0.202(0.006)$ & $0.871(0.049)$ & $0.200(0.008)$ & $0.880(0.037)$ \\
\hline LGBM & $0.204(0.012)$ & $0.871(0.074)$ & $0.197(0.012)$ & $0.861(0.066)$ \\
\hline
\end{tabular}

${ }^{\mathrm{a}} \mathrm{KNN}$ : k-nearest neighbors.

${ }^{\mathrm{b}} \mathrm{DT}$ : decision tree.

${ }^{\mathrm{c}}$ Best-performing models.

${ }^{\mathrm{d}} \mathrm{RF}$ : random forest.

eXGB: XGBoost.

${ }^{\mathrm{f}} \mathrm{LR}$ : logistic regression.

${ }^{\mathrm{g}}$ MLP: multilayer perceptron.

${ }^{\mathrm{h}}$ LGBM: LightGBM.

The predictability of ICU needs is less satisfactory than hospitalization needs, particularly for the prehospitalization stage. We hypothesize that this difficulty was partially related to the smaller number of individuals with ICU internments, together with the presence of missing values associated with ICU internment needs for most individuals. Even though we can achieve recall levels over $90 \%$ with gradient boosting (XGBoost) in a posthospitalization setting, it comes at the cost of a considerably low precision (with one-third of predictions seen in practice). Still, the best-performing predictive models are suggested to support monitoring decisions at the hospital bedside, as their recall and specificity are considerably high.

\section{Respiratory Support}

Figure 8 and Table 4 assess respiratory assistance needs for hospitalized individuals with SARS-CoV-2, considering three assistance modes: (1) ventilation support, (2) oxygen therapy, and (3) combined ventilation and oxygen therapies.
Demographic, comorbidity, and time-to-hospitalization factors were used as input variables.

Individuals without a SARS-CoV-2-negative test result after infection were excluded from this analysis. As respiratory support is a multiclass variable, we considered a different performance evaluation by focusing on (1) the recall for each major class (ventilation, oxygen, and nonrequired support), (2) the precision of individuals with oxygen or ventilation assistance, and (3) the Cohen kappa coefficient.

XGBoost, LightGBM, and random forests attained a satisfactory identification of hospitalized individuals who may require respiratory support in the future, generally providing recalls for each assistance mode around $60 \%$ at the cost of a $40 \%$ precision. According to the conducted methodology, they are thus pinpointed as good candidates to support in-hospital care decisions. 
Figure 8. Predictability of respiratory support needs—assisted ventilation, oxygen therapy, and combined support—for hospitalized individuals with SARS-CoV-2. Performance of the best F1 predictor (right) and recall-oriented predictor (left) is shown. XGB: XGBoost, LGBM: LightGBM.

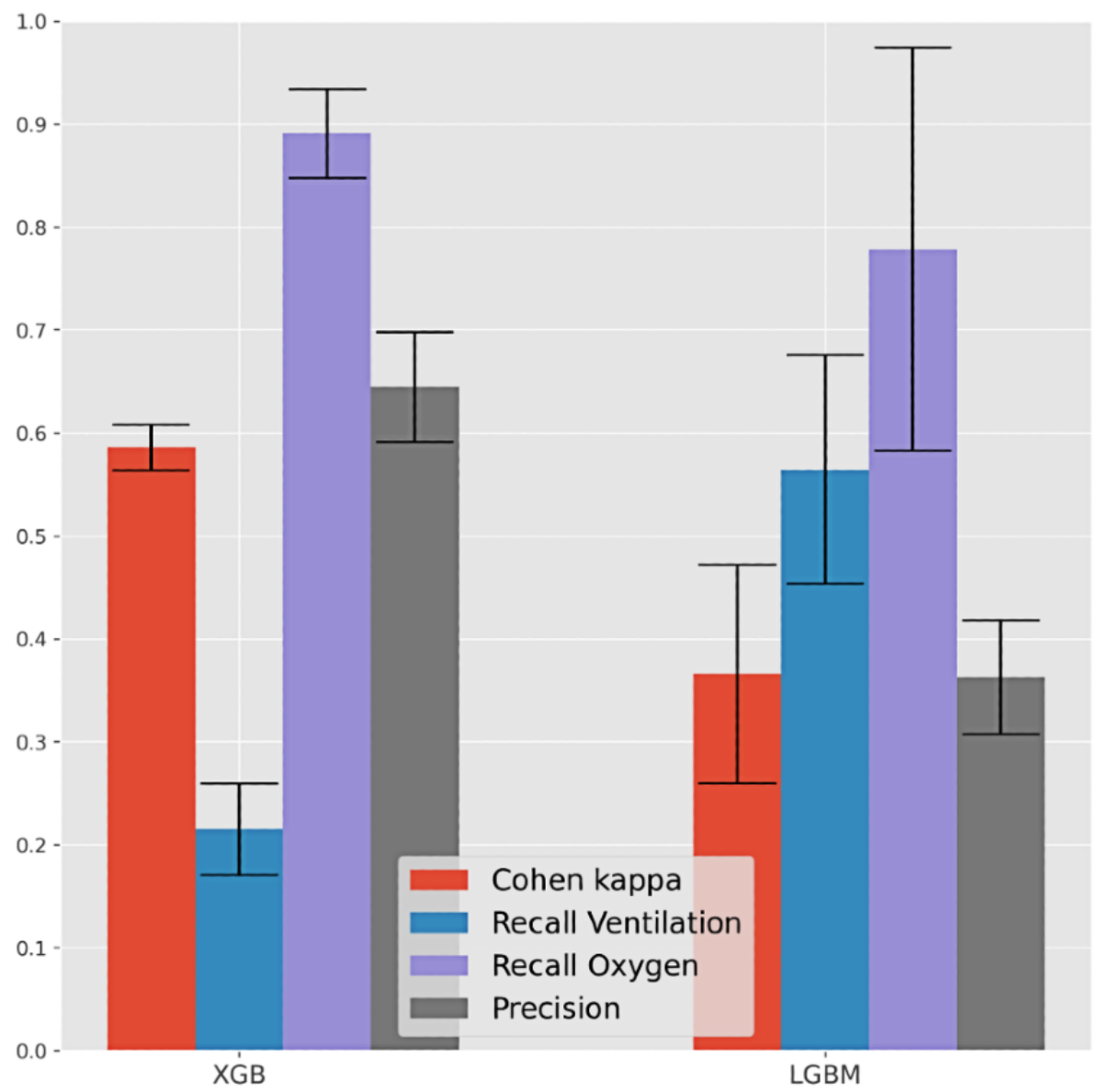

Table 4. Predictability of respiratory needs per predictive model.

\begin{tabular}{lll}
\hline Model & Kappa optimization, kappa, mean (SD) & Recalls optimization average, kappa, mean (SD) \\
\hline $\mathrm{KNN}^{\mathrm{a}}$ & $0.324(0.050)$ & $0.162(0.022)$ \\
$\mathrm{DT}^{\mathrm{b}}$ & $0.708(0.017)^{\mathrm{c}}$ & $0.110(0.126)$ \\
$\mathrm{RF}^{\mathrm{d}}$ & $0.518(0.013)$ & $0.017(0.014)$ \\
$\mathrm{XGB}^{\mathrm{e}}$ & $0.586(0.022)$ & $0.043(0.013)$ \\
$\mathrm{LR}^{\mathrm{f}}$ & $0.080(0.009)$ & $0.070(0.009)$ \\
$\mathrm{MLP}^{\mathrm{g}}$ & $0.464(0.046)$ & $0.204(0.164)$ \\
$\mathrm{LGBM}^{\mathrm{h}}$ & $0.567(0.037)$ & $0.366(0.106)^{\mathrm{c}}$ \\
\hline
\end{tabular}

${ }^{\mathrm{a}} \mathrm{KNN}$ : k-nearest neighbors.

${ }^{\mathrm{b}} \mathrm{DT}$ : decision tree.

${ }^{\mathrm{c}}$ Best-performing models.

${ }^{\mathrm{d}} \mathrm{RF}$ : random forest.

${ }^{\mathrm{e}} \mathrm{XGB}$ : XGBoost.

${ }^{\mathrm{f}} \mathrm{LR}$ : logistic regression.

${ }^{\mathrm{g}}$ MLP: multilayer perceptron.

h LGBM: LightGBM. 


\section{Survivability (Outcome)}

Finally, Figures 9 and 10 and Table 5 provide an analysis of the ability of the models to predict recovery-or-death outcomes for individuals with SARS-CoV-2 infection at three time points: (1) before hospitalization (at the time of testing), (2) after hospitalization, and (3) after ICU admission when applicable. To this end, we preserved the input variables and validation methodology (see Methods section) considered in previous scenarios.
Our results showed a high ability to identify death outcomes. However, at the SARS-CoV-2 testing stage, this comes at a cost of incorrectly classifying two-thirds of individuals susceptible to death. In the posthospitalization scenario, we achieved more balanced results, with both precision and recall around $75 \%$ using gradient boosting (XGBoost and LightGBM). The introduction of the intensive care variable hampered the results since it restricted the analysis of deaths to individuals with acute needs and dependent on continuous care instruments.

Figure 9. Predictability of the survivability (outcome) of infected individuals at 3 stages. Results for the best F1 predictor (left) and recall-optimized predictor (right) per stage are shown. XGB: XGBoost, LGBM: LightGBM.

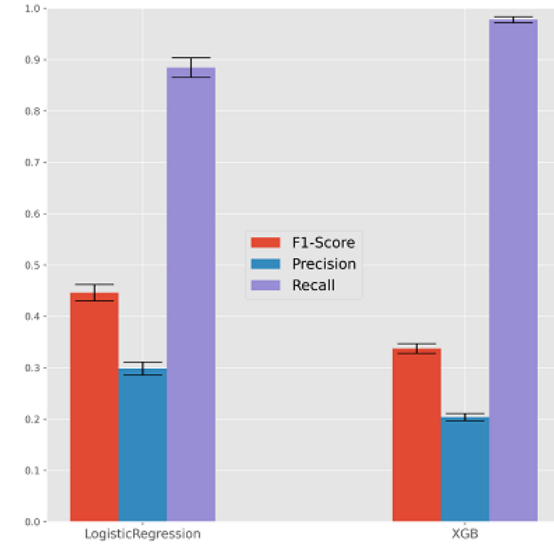

(A) before hospitalization

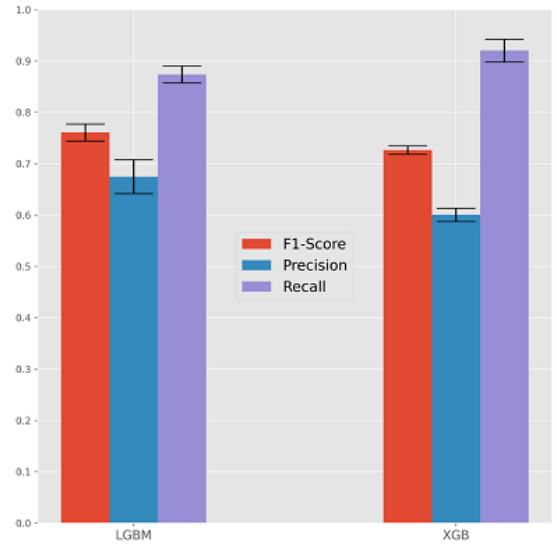

(B) after hospitalization

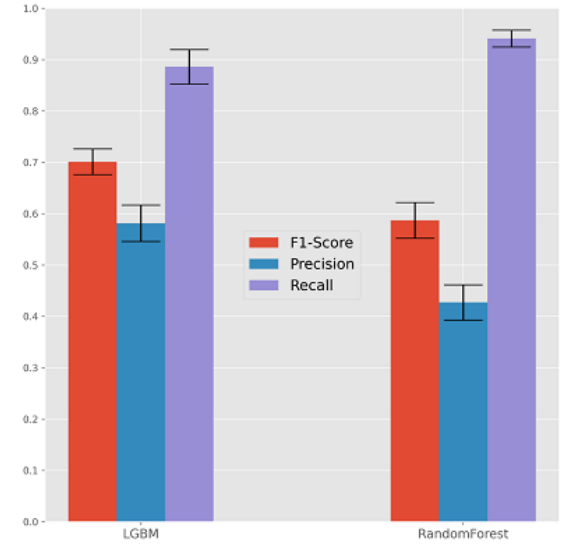

(C) after ICU interment

Figure 10. Receiver operating characteristic curves with the predictive behavior of the selected classifiers in asserting patient survivability at the time of hospitalization. XGB: XGBoost, MLP: multilayer perceptron, LGBM: LightGBM.

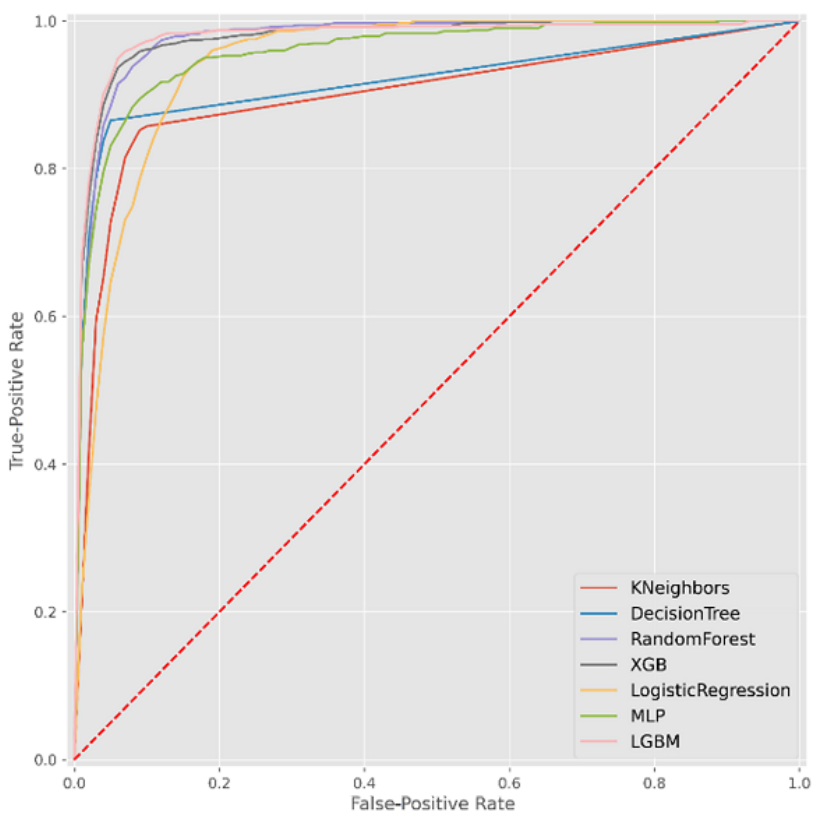

(A) F1 optimization

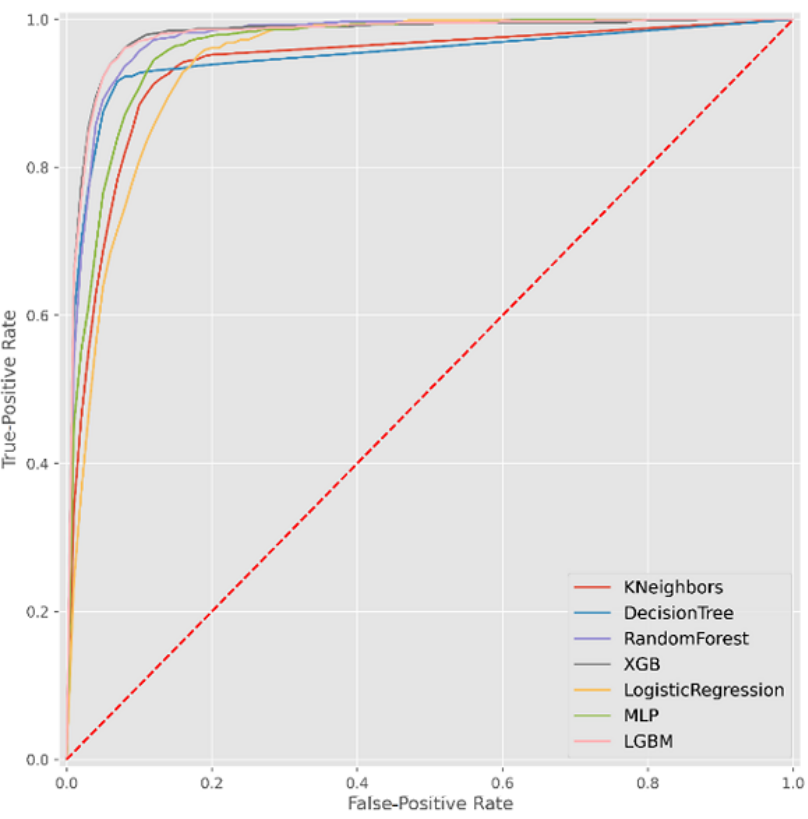

(B) $F \beta=2$ (recall-oriented) optimization 
Table 5. Predictability of survivability per predictive model.

\begin{tabular}{|c|c|c|c|c|}
\hline \multirow[t]{2}{*}{ Model } & \multicolumn{2}{|c|}{ F1 optimization, mean (SD) } & \multicolumn{2}{|c|}{$\mathrm{F}_{\beta=2}$ (recall-oriented) optimization, mean (SD) } \\
\hline & F1-score & Recall & F1-score & Recall \\
\hline \multicolumn{5}{|c|}{ At the time of hospitalization } \\
\hline $\mathrm{KNN}^{\mathrm{a}}$ & $0.616(0.035)$ & $0.735(0.048)$ & $0.546(0.021)$ & $0.901(0.030)$ \\
\hline $\mathrm{DT}^{\mathrm{b}}$ & $0.707(0.013)$ & $0.864(0.011)$ & $0.673(0.022)$ & $0.908(0.017)$ \\
\hline $\mathrm{RF}^{\mathrm{c}}$ & $0.696(0.030)$ & $0.901(0.030)$ & $0.666(0.021)$ & $0.666(0.021)$ \\
\hline $\mathrm{XGB}^{\mathrm{d}}$ & $0.765(0.025)$ & $0.834(0.042)$ & $0.726(0.008)$ & $0.920(0.022)$ \\
\hline $\mathrm{LR}^{\mathrm{e}}$ & $0.492(0.012)$ & $0.909(0.019)$ & $0.476(0.035)$ & $0.916(0.022)$ \\
\hline$M L P^{f}$ & $0.681(0.024)$ & $0.824(0.027)$ & $0.569(0.020)$ & $0.922(0.023)$ \\
\hline $\mathrm{LGBM}^{\mathrm{g}}$ & $0.761(0.017)$ & $0.874(0.016)$ & $0.717(0.036)$ & $0.922(0.021)$ \\
\hline \multicolumn{5}{|c|}{ At the time of intensive care unit admission } \\
\hline $\mathrm{KNN}$ & $0.582(0.040)$ & $0.740(0.053)$ & $0.527(0.030)$ & $0.885(0.049)$ \\
\hline DT & $0.652(0.045)$ & $0.879(0.035)$ & $0.638(0.032)$ & $0.922(0.023)$ \\
\hline $\mathrm{RF}$ & $0.630(0.018)$ & $0.908(0.039)$ & $0.587(0.035)$ & $0.941(0.016)$ \\
\hline $\mathrm{XGB}$ & $0.703(0.035)$ & $0.838(0.068)$ & $0.672(0.021)$ & $0.918(0.051)$ \\
\hline LR & $0.497(0.018)$ & $0.908(0.031)$ & $0.470(0.049)$ & $0.920(0.028)$ \\
\hline MLP & $0.633(0.044)$ & $0.790(0.094)$ & $0.529(0.019)$ & $0.935(0.020)$ \\
\hline LGBM & $0.701(0.025)$ & $0.886(0.034)$ & $0.672(0.024)$ & $0.915(0.027)$ \\
\hline
\end{tabular}

${ }^{\mathrm{a}} \mathrm{KNN}$ : k-nearest neighbors.

${ }^{\mathrm{b}} \mathrm{DT}$ : decision tree.

${ }^{\mathrm{c}} \mathrm{RF}$ : random forest.

${ }^{\mathrm{d}}$ XGB: XGBoost.

${ }^{\mathrm{e}}$ LR: logistic regression.

${ }^{\mathrm{f}}$ MLP: multilayer perceptron.

${ }^{\mathrm{g}}$ LGBM: LightGBM.

\section{Determinants of Predictability}

To assess the determinant factors underlying the achieved predictability levels, we first statistically tested the correlation between input and output variables using chi-square tests, ANOVA (analysis of variance), and their nonparametric counterparts, yielding results similar to those by Nogueira et al [33]. For a more in-depth understanding of the feature relevance for the assessed predictive models, Figures 11 and 12 illustrate the importance of the top features. To this end, we considered relevance outputs from gradient boosting (XGBoost) due to its competitively high performance across all outcomes, as well as the logistic regression for the hospitalization outcome by computing the Wald statistic to assess the significance of the coefficients for predictions. 
Figure 11. Top features and their importance for each target variable at the time of SARS-CoV-2 testing (prehospitalization). ICU: intensive care unit, LR: logistic regression.

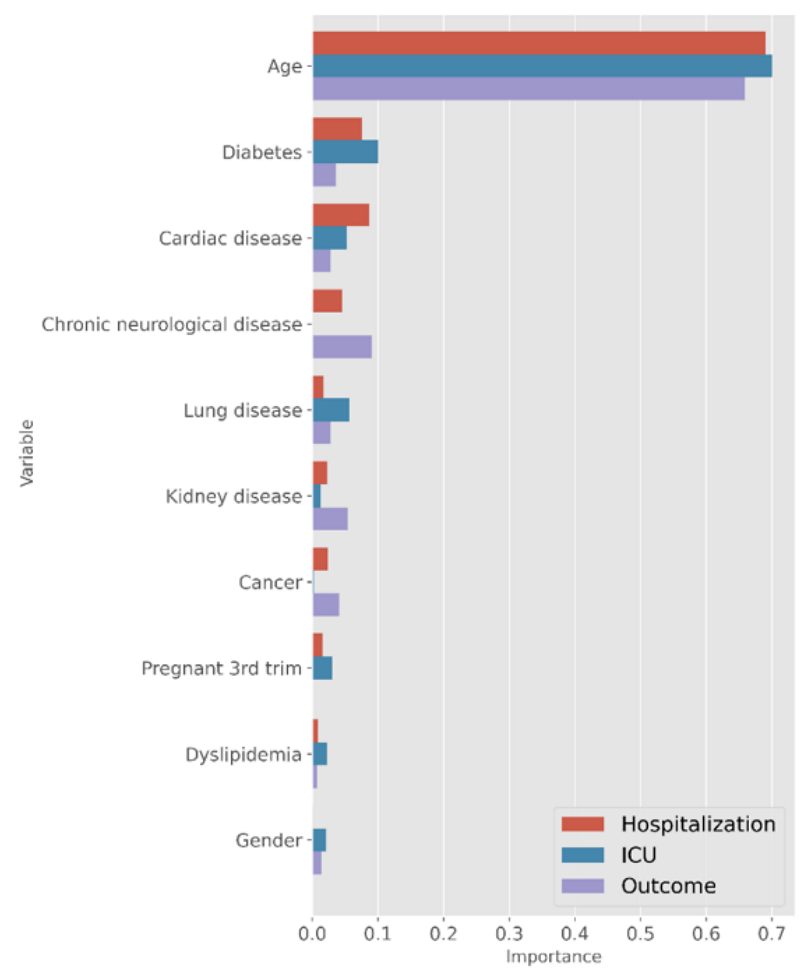

(A) XGBoost importance

We can observe that XGBoost distinguishes the relevance of different comorbidities for the target variables along each stage of the care process. In addition to the age variable, the onset period to hospitalization in days was also found to be a critical factor affecting the decisions (Figure 12). The high relevance

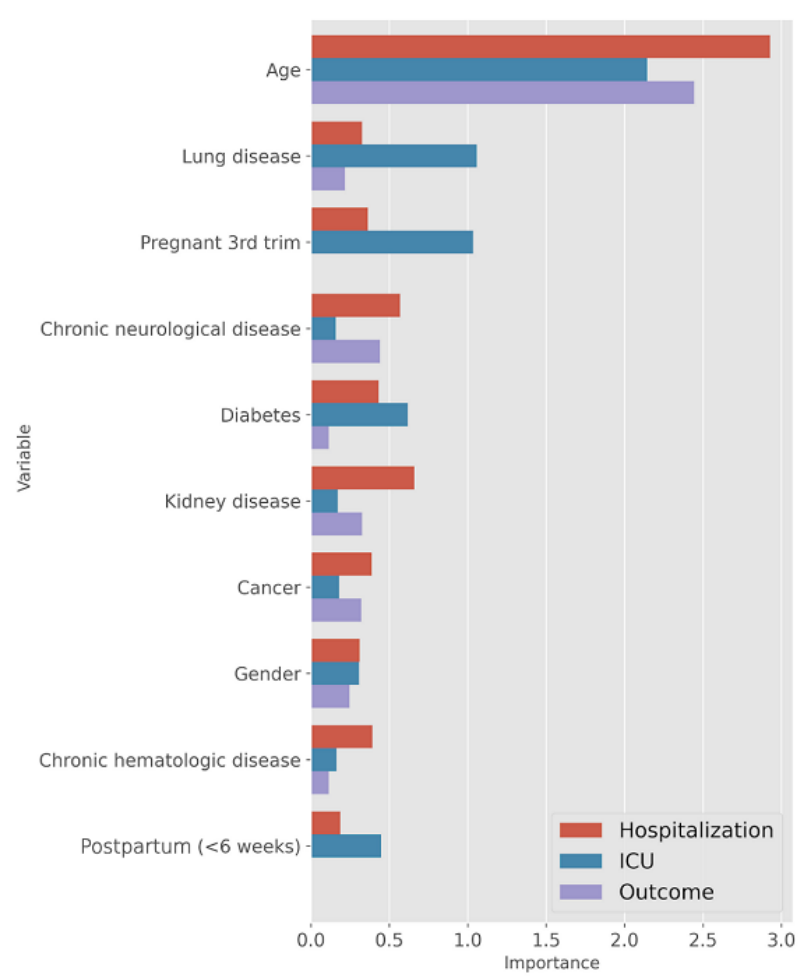

(B) LR importance (Wald statistic)

of this variable consistently had top rank among associative models-XGBoost, random forests, and decision trees-pinpointing the importance of its collection for computer-aided predictions of ICU internment and respiratory needs.

Figure 12. XGBoost top features and their importance for the different outcomes at posthospitalization. ICU: intensive care unit.

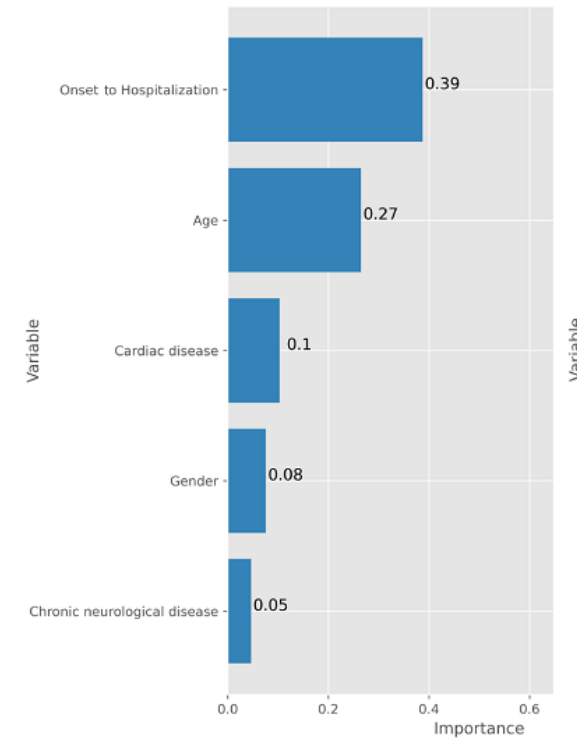

(A) ICU admission

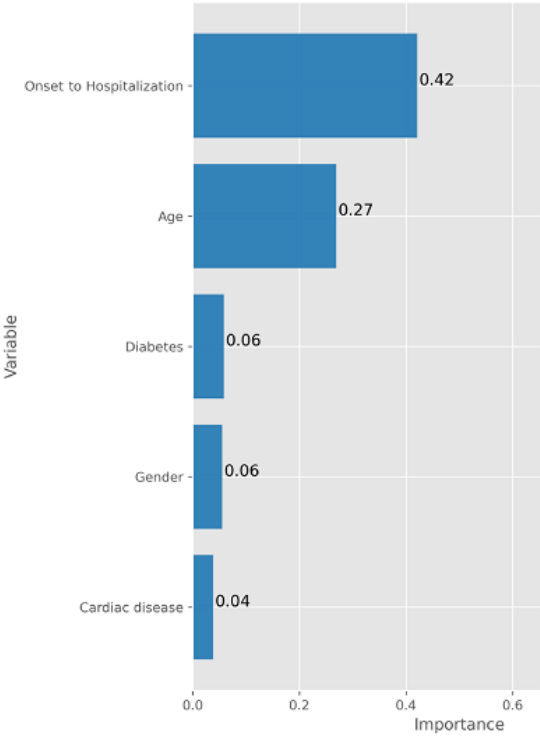

(B) respiratory needs

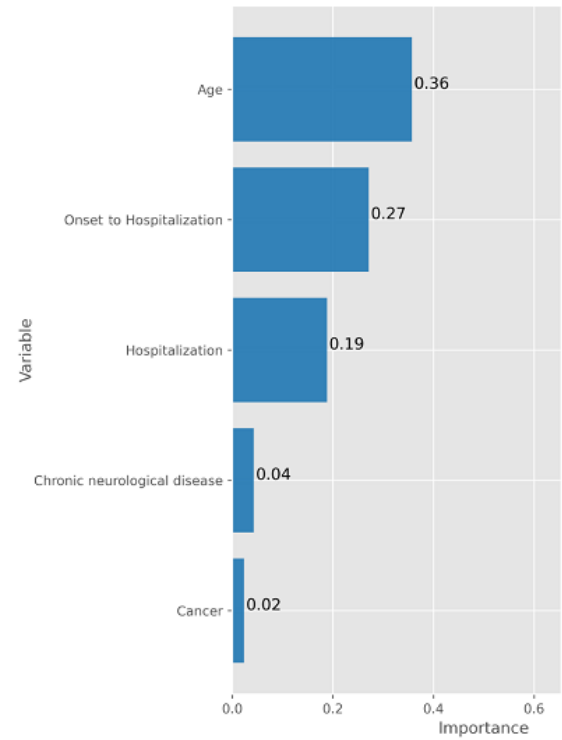

(C) survivability
Complementarily, Figure 13 offers additional insights into the target predictive tasks by plotting some of the characteristics of the correctly classified individuals against incorrectly classified individuals with XGBoost. Particular attention should be paid to the differences between true positives and false negatives, that is, to the individuals requiring care, in order to guarantee their timely and proper assistance. The susceptibility 
to false negatives is higher for individuals within the 40-60-year age category and without comorbidities.

Figure 13. The characteristics of incorrectly predicted individuals with XGBoost. Particular attention should be paid to the differences between true-positive and false-negative individuals given their clinical relevance. ICU: intensive care unit.
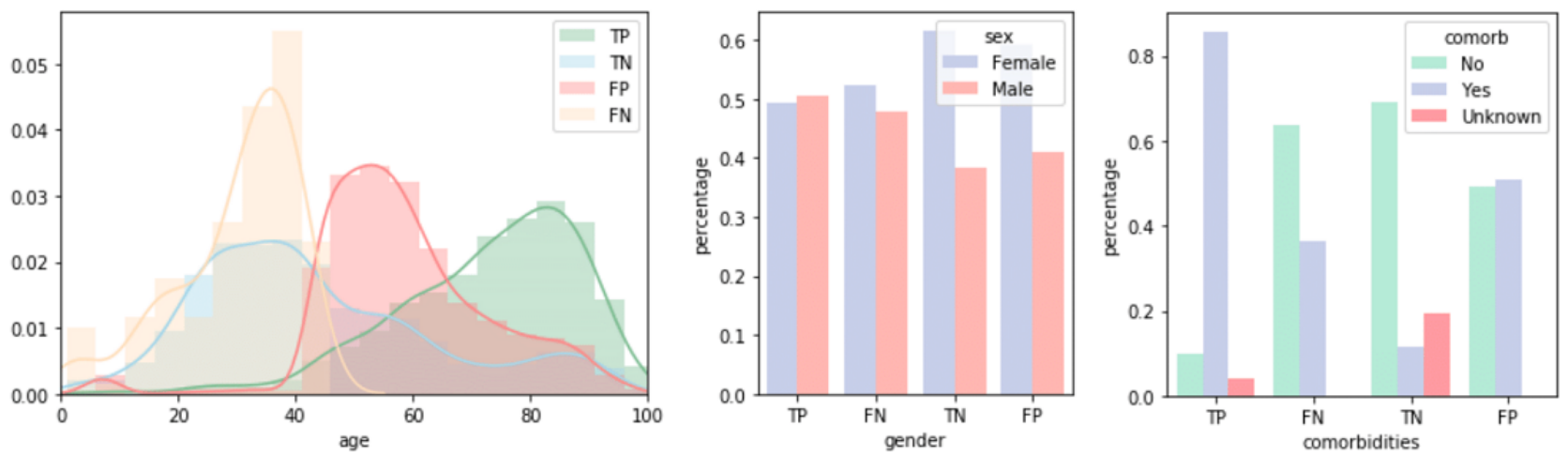

(A) Hospitalizations: profile of false-positive (FP) and false-negative (FN) individuals
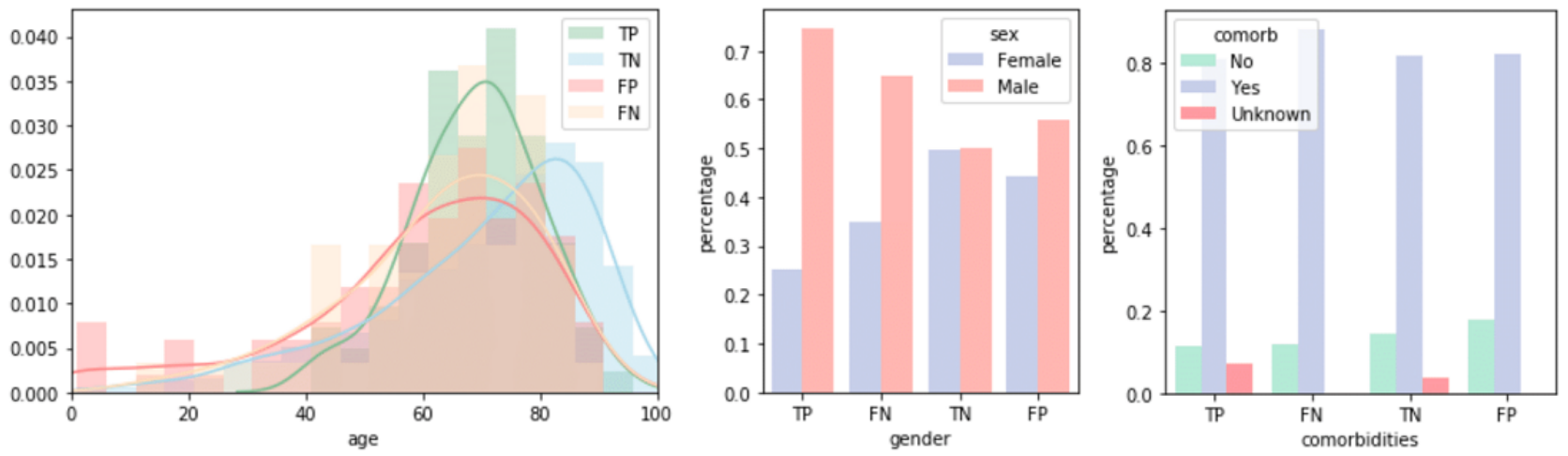

(B) ICU admissions (posthospitalization): profile of false-positive (FP) and false-negative (FN) individuals
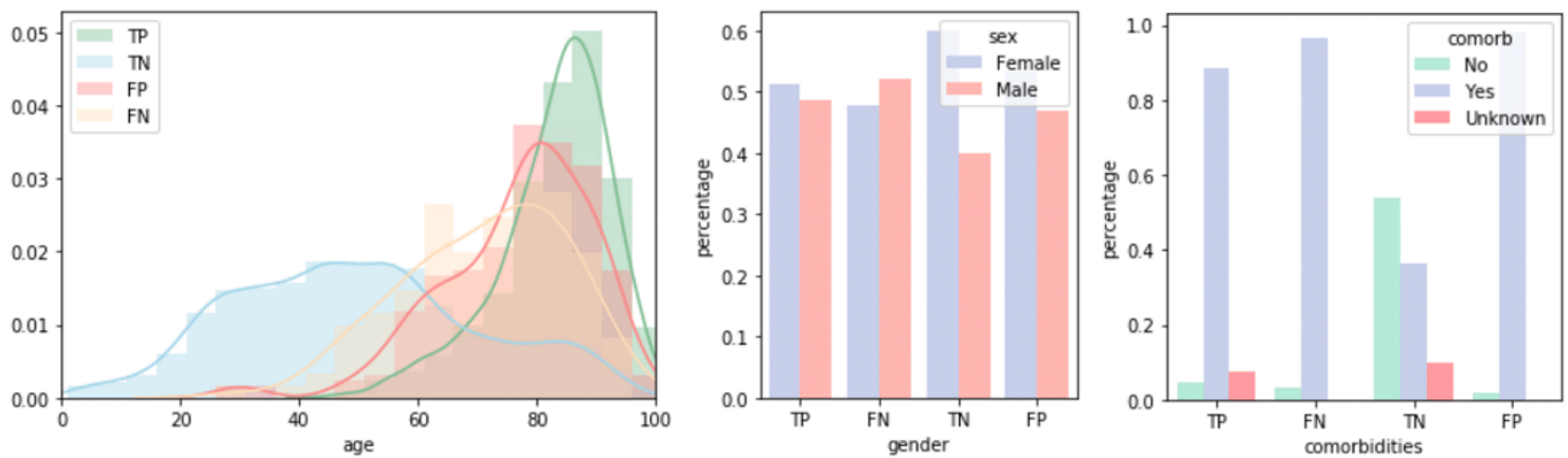

(C) Survivability: profile of false-positive (FP) and false-negative (FN) individuals

\section{Clinical Decision Support System}

The learned predictive models based on simple variables (stage, age, gender, and comorbidities) have been made available to health care providers within a recommendation system with graphical facilities. The serialized predictive models are used for the efficient testing of individuals at the different stages of the care cycle (testing, hospitalization, ICU admission) for the different outcome variables (care needs) after inserting essential demographic and comorbidity features. The output provides a bounded statistic based on the estimation returned by the predictive models achieving better recall and F1-measure for each outcome variable. Figure 14 provides a visualization of the graphical interface. 
Figure 14. Snapshot of the provided clinical decision support system.
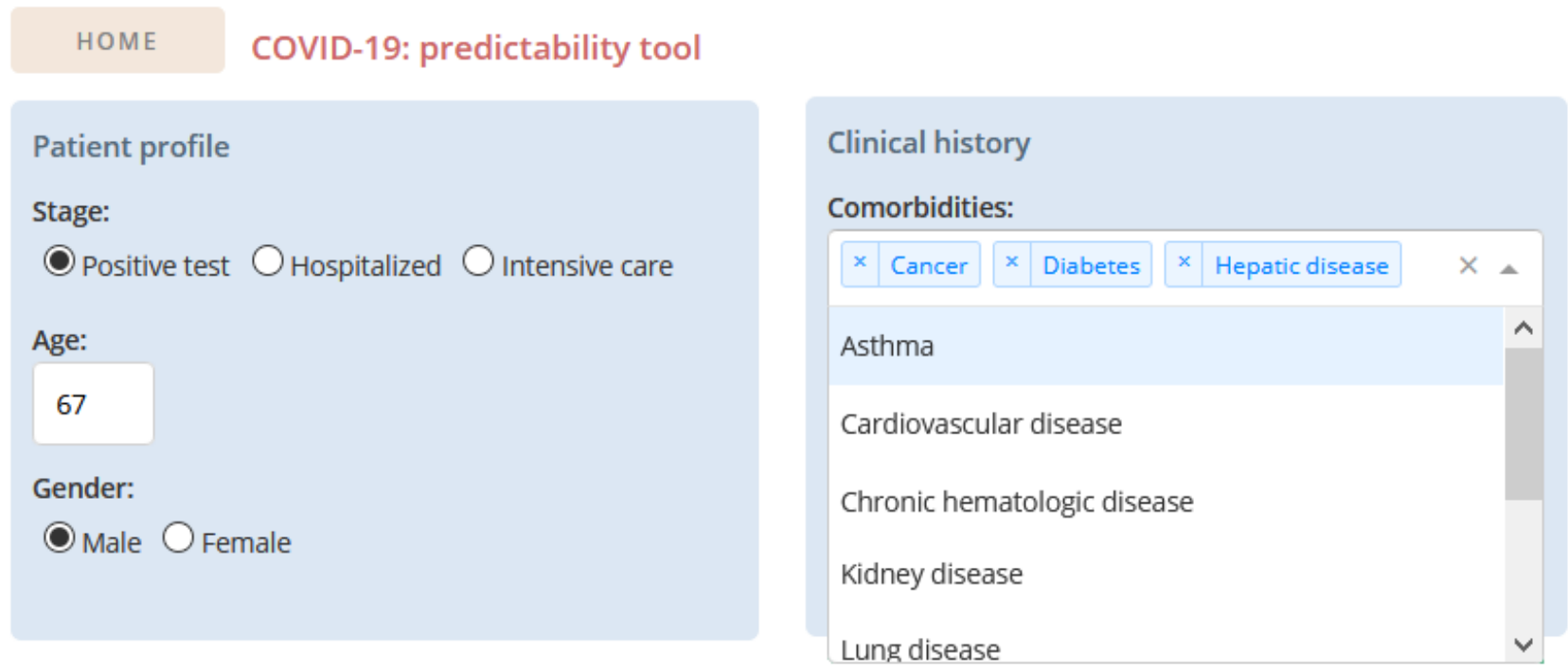

\section{RUN QUERY}

Result:

Input the patient profile...

The variables required for each outcome score calculation are usually available at hospitals, and the tool is easy to use. Although recommendations are provided within a statistical frame, the tool does not categorize the risk into low- or high-risk patients as clinical experts are more informed to approximate this risk. In addition, we advise caution for clinicians who intend to use this tool as a predictive guide, especially for survivability analysis. Clinicians must balance the predictions from this tool against their practical experience.

In collaboration with DGS, our predictors are expected to be provided within public hospitals and care contact centers of the Portuguese Health Service (Serviço Nacional de Saúde), particularly to support remote care monitoring decisions.

The decision support system is available as a software tool on GitHub [34].

\section{Discussion}

\section{Principal Findings}

This work offers a discussion on the predictability of hospitalization needs, ICU admissions, respiratory assistance, and survivability outcome in individuals infected with SARS-CoV-2 in Portugal as of June 30, 2020. A retrospective cohort with all confirmed COVID-19 cases since March, encompassing demographic and comorbidity variables, was considered as the target population in this study.

The results for the given cohort reveal that (1) over $75 \%$ of hospitalization needs can be identified at the time of SARS-CoV-2 testing (with $>50 \%$ precision); (2) ICU needs are generally less predictable at both the pre- and posthospitalization stages in the given cohort; (3) respiratory assistance needs (including ventilation support, oxygen therapy, and combined ventilation-oxygen support) achieved recall levels above $60 \%$ (with $>50 \%$ precision); (4) death risk along different stages (testing time, after hospitalization, and after ICU admission) had the highest degree of predictability.

The predictive models yielding better accuracy performance were associative classifiers, particularly XGBoost and RandomForests, neural networks with hyperparameterized architectures, and logistic regressors, with the optimal choice varying in accordance with the target variable and evaluation measure.

Publications on COVID-19 using machine learning models for different outcomes have been rapidly increasing. Gao et al [35] developed a model that includes the mortality risk prediction and reported an F1 ranging from 0.65 to 0.69 ( $\mathrm{K}=0.61-0.65)$, in line with our findings. Alternative studies [28,36,37] offer additional results for generalizing results and identifying population-specific differences. Yet, most of these studies do not comprehensively assess models' performance or the cohort characteristics, impeding solid cross-population findings.

\section{Limitations}

This study has some inherent shortcomings that should be noted: (1) the number of clinical variables for the outcomes of interest were limited (eg, BMI and clinical symptoms were missing); (2) further external validation of the selected models is required; and (3) although some inconsistencies (listed in the Cohort Description section) and missing/unknown entries in the original DGS data set were excluded, data acquisition problems may still persist and influence the outcomes of this work. The fully autonomous and parameter-free nature of the proposed computational approach/models allows it to be dynamically retrained with updated data. 


\section{Concluding Remarks}

In this work, we developed a web-based clinical decision support tool without biological variables as input that can be used by clinicians. The conducted work pinpoints the relevance of the proposed predictive models to aid medical decisions for the
Portuguese population, including both remote monitoring and in-hospital care decisions. Predicting the most probable outcomes along the life cycle of a SARS-CoV-2-infected individual can identify patients who are expected to develop severe illness, thus optimizing the allocation of health care resources and supporting more vulnerable patients.

\section{Acknowledgments}

The authors thank Portuguese Directorate General of Health (DGS) for providing the data. Data are available upon reasonable request. This work was supported by Fundação para a Ciência e a Tecnologia (FCT), through IDMEC, under LAETA project (UIDB/50022/2020), IPOscore (DSAIPA/DS/0042/2018) and Data2Help (DSAIPA/AI/ 0044/2018) projects, the contract CEECIND/01399/2017 to RSC, FCT/MCTES funds for INESC-ID (UIDB/50021/2020) and the Associate Laboratory for Green Chemistry - LAQV (UIDB/50006/2020 and UIDP/50006/2020).

\section{Conflicts of Interest}

None declared.

\section{Multimedia Appendix 1}

Optimized parameters for the best-performing classifiers (Figures 4, 6, 8, and 9).

[PNG File, 71 KB-Multimedia Appendix 1]

\section{References}

1. Chan J, Yuan S, Kok K, To K, Chu H, Yang J, et al. A familial cluster of pneumonia associated with the 2019 novel coronavirus indicating person-to-person transmission: a study of a family cluster. The Lancet 2020 Feb;395(10223):514-523. [doi: 10.1016/s0140-6736(20)30154-9]

2. Zhu N, Zhang D, Wang W, Li X, Yang B, Song J, et al. A Novel Coronavirus from Patients with Pneumonia in China, 2019. N Engl J Med 2020 Feb 20;382(8):727-733. [doi: 10.1056/nejmoa2001017]

3. Fu L, Wang B, Yuan T, Chen X, Ao Y, Fitzpatrick T, et al. Clinical characteristics of coronavirus disease 2019 (COVID-19) in China: A systematic review and meta-analysis. J Infect 2020 Jun;80(6):656-665 [FREE Full text] [doi: 10.1016/j.jinf.2020.03.041] [Medline: 32283155]

4. World Health Organization. URL: https://www.who.int [accessed 2020-09-01]

5. Singh AK, Gupta R, Ghosh A, Misra A. Diabetes in COVID-19: Prevalence, pathophysiology, prognosis and practical considerations. Diabetes Metab Syndr 2020 Jul;14(4):303-310 [FREE Full text] [doi: 10.1016/j.dsx.2020.04.004] [Medline: 32298981]

6. Sanyaolu A, Okorie C, Marinkovic A, Patidar R, Younis K, Desai P, et al. Comorbidity and its Impact on Patients with COVID-19. SN Compr Clin Med 2020 Jun 25;2(8):1-8 [FREE Full text] [doi: 10.1007/s42399-020-00363-4] [Medline: 32838147]

7. Wang Y, Lu X, Li Y, Chen H, Chen T, Su N, et al. Clinical Course and Outcomes of 344 Intensive Care Patients with COVID-19. Am J Respir Crit Care Med 2020 Jun 01;201(11):1430-1434. [doi: 10.1164/rccm.202003-0736le]

8. Guan W, Liang W, Zhao Y, Liang H, Chen Z, Li Y, China Medical Treatment Expert Group for COVID-19. Comorbidity and its impact on 1590 patients with COVID-19 in China: a nationwide analysis. Eur Respir J 2020 May 26;55(5):2000547 [FREE Full text] [doi: 10.1183/13993003.00547-2020] [Medline: 32217650]

9. Du RH, Liang LR, Yang CQ, Wang W, Cao TZ, Li M, et al. Predictors of mortality for patients with COVID-19 pneumonia caused by SARS-CoV-2: a prospective cohort study. Eur Respir J 2020 Sep 24;56(3):2050524. [doi: 10.1183/13993003.50524-2020] [Medline: 32973076]

10. Shi Y, Yu X, Zhao H, Wang H, Zhao R, Sheng J. Host susceptibility to severe COVID-19 and establishment of a host risk score: findings of 487 cases outside Wuhan. Crit Care 2020 Mar 18;24(1):108 [FREE Full text] [doi: 10.1186/s13054-020-2833-7] [Medline: 32188484 ]

11. Hu H, Yao N, Qiu Y. Comparing Rapid Scoring Systems in Mortality Prediction of Critically Ill Patients With Novel Coronavirus Disease. Acad Emerg Med 2020 Jun 21;27(6):461-468 [FREE Full text] [doi: 10.1111/acem.13992] [Medline: $\underline{32311790]}$

12. Cho S, Park S, Song M, Bae YY, Lee D, Kim D. Prognosis Score System to Predict Survival for COVID-19 Cases: a Korean Nationwide Cohort Study. J Med Internet Res 2021 Feb 22;23(2):e26257 [FREE Full text] [doi: 10.2196/26257] [Medline: 33539312]

13. Agosto A, Giudici P. A Poisson Autoregressive Model to Understand COVID-19 Contagion Dynamics. SSRN Journal Preprint posted online Mar 10, 2020. [doi: 10.2139/ssrn.3551626] 
14. Li X, Xu S, Yu M, Wang K, Tao Y, Zhou Y, et al. Risk factors for severity and mortality in adult COVID-19 inpatients in Wuhan. J Allergy Clin Immunol 2020 Jul;146(1):110-118 [FREE Full text] [doi: 10.1016/j.jaci.2020.04.006] [Medline: $\underline{32294485}$

15. Barman RK, Mukhopadhyay A, Maulik U, Das S. Identification of infectious disease-associated host genes using machine learning techniques. BMC Bioinformatics 2019 Dec 27;20(1):736 [FREE Full text] [doi: 10.1186/s 12859-019-3317-0] [Medline: 31881961$]$

16. Luz C, Vollmer M, Decruyenaere J, Nijsten M, Glasner C, Sinha B. Machine learning in infection management using routine electronic health records: tools, techniques, and reporting of future technologies. Clin Microbiol Infect 2020 Oct;26(10):1291-1299. [doi: 10.1016/j.cmi.2020.02.003] [Medline: 32061798]

17. Boutilier J, Chan T, Ranjan M, Deo S. Risk Stratification for Early Detection of Diabetes and Hypertension in Resource-Limited Settings: Machine Learning Analysis. J Med Internet Res 2021 Jan 21;23(1):e20123 [FREE Full text] [doi: 10.2196/20123] [Medline: 33475518]

18. Sujath R, Chatterjee JM, Hassanien AE. A machine learning forecasting model for COVID-19 pandemic in India. Stoch Environ Res Risk Assess 2020 May 30;34(7):959-972. [doi: 10.1007/s00477-020-01827-8]

19. Chassagnon G, Vakalopoulou M, Battistella E, Christodoulidis S, Hoang-Thi T, Dangeard S, et al. AI-driven quantification, staging and outcome prediction of COVID-19 pneumonia. Med Image Anal 2021 Jan;67:101860 [FREE Full text] [doi: 10.1016/j.media.2020.101860] [Medline: 33171345]

20. Assaf D, Gutman Y, Neuman Y, Segal G, Amit S, Gefen-Halevi S, et al. Utilization of machine-learning models to accurately predict the risk for critical COVID-19. Intern Emerg Med 2020 Nov 18;15(8):1435-1443 [FREE Full text] [doi: 10.1007/s11739-020-02475-0] [Medline: 32812204]

21. Wu G, Yang P, Xie Y, Woodruff HC, Rao X, Guiot J, et al. Development of a clinical decision support system for severity risk prediction and triage of COVID-19 patients at hospital admission: an international multicentre study. Eur Respir J 2020 Aug 02;56(2):2001104 [FREE Full text] [doi: 10.1183/13993003.01104-2020] [Medline: 32616597]

22. Wynants L, Van Calster B, Collins GS, Riley RD, Heinze G, Schuit E, et al. Prediction models for diagnosis and prognosis of covid-19 infection: systematic review and critical appraisal. BMJ 2020 Apr 07;369:m1328 [FREE Full text] [doi: 10.1136/bmj.m1328] [Medline: $\underline{\text { 32265220] }}$

23. Burdick H, Lam C, Mataraso S, Siefkas A, Braden G, Dellinger RP, et al. Prediction of respiratory decompensation in Covid-19 patients using machine learning: The READY trial. Comput Biol Med 2020 Sep;124:103949 [FREE Full text] [doi: 10.1016/j.compbiomed.2020.103949] [Medline: 32798922]

24. Liang W, Liang H, Ou L, Chen B, Chen A, Li C, China Medical Treatment Expert Group for COVID-19. Development and Validation of a Clinical Risk Score to Predict the Occurrence of Critical Illness in Hospitalized Patients With COVID-19. JAMA Intern Med 2020 Aug 01;180(8):1081-1089 [FRE Full text] [doi: 10.1001/jamainternmed.2020.2033] [Medline: 32396163]

25. Gayam V, Chobufo MD, Merghani MA, Lamichhane S, Garlapati PR, Adler MK. Clinical characteristics and predictors of mortality in African-Americans with COVID-19 from an inner-city community teaching hospital in New York. J Med Virol 2021 Feb 05;93(2):812-819 [FREE Full text] [doi: 10.1002/jmv.26306] [Medline: 32672844]

26. Núñez-Gil IJ, Fernández-Pérez C, Estrada V, Becerra-Muñoz VM, El-Battrawy I, Uribarri A, HOPE COVID-19 Investigators. Mortality risk assessment in Spain and Italy, insights of the HOPE COVID-19 registry. Intern Emerg Med 2020 Nov 09;51(1):e13436 [FREE Full text] [doi: 10.1007/s11739-020-02543-5] [Medline: 33165755]

27. Iwendi C, Bashir AK, Peshkar A, Sujatha R, Chatterjee JM, Pasupuleti S, et al. COVID-19 Patient Health Prediction Using Boosted Random Forest Algorithm. Front Public Health 2020 Jul 3;8:357 [FREE Full text] [doi: 10.3389/fpubh.2020.00357] [Medline: $\underline{32719767]}$

28. Vaid A, Somani S, Russak AJ, De Freitas JK, Chaudhry FF, Paranjpe I, et al. Machine Learning to Predict Mortality and Critical Events in a Cohort of Patients With COVID-19 in New York City: Model Development and Validation. J Med Internet Res 2020 Nov 06;22(11):e24018 [FREE Full text] [doi: 10.2196/24018] [Medline: 33027032]

29. Snoek J, Larochelle H, Adams RP. Practical Bayesian optimization of machine learning algorithms. In: NIPS'12: Proceedings of the 25th International Conference on Neural Information Processing Systems. Red Hook, NY: Curran Associates Inc; 2012 Presented at: International Conference on Neural Information Processing Systems; Dec 3-6; Nevada p. 2951-2959 URL: https://papers.nips.cc/paper/2012/file/05311655a15b75fab86956663e1819cd-Paper.pdf

30. Pedregosa F, Varoquaux G, Gramfort A, Michel V, Thirion B, Grisel O, et al. Scikit-learn: Machine learning in Python. J Mach Learn Res 2011;12:2825-2830 [FREE Full text]

31. Chen T, Guestrin C. XGBoost: A scalable tree boosting system. In: Proceedings of the 22nd ACM SIGKDD International Conference on Knowledge Discovery and Data Mining. 2016 Presented at: KDD '16: The 22nd ACM SIGKDD International Conference on Knowledge Discovery and Data Mining; Aug 13-16; San Francisco, CA p. 785-794. [doi: 10.1145/2939672.2939785]

32. Plotly. URL: https://plotly.com/dash/ [accessed 2021-04-13]

33. Nogueira PJ, de Araújo Nobre M, Costa A, Ribeiro RM, Furtado C, Bacelar Nicolau L, et al. The Role of Health Preconditions on COVID-19 Deaths in Portugal: Evidence from Surveillance Data of the First 20293 Infection Cases. J Clin Med 2020 Jul 24;9(8):3442 [FREE Full text] [doi: 10.3390/jcm9082368] [Medline: 32722159] 
34. GitHub. URL: https://github.com/Andrempp/COVIDCalculator [accessed 2021-04-13]

35. Gao Y, Cai G, Fang W, Li H, Wang S, Chen L, et al. Machine learning based early warning system enables accurate mortality risk prediction for COVID-19. Nat Commun 2020 Oct 06;11(1):5033 [FREE Full text] [doi: 10.1038/s41467-020-18684-2] [Medline: 33024092]

36. Hu C, Liu Z, Jiang Y, Shi O, Zhang X, Xu K, et al. Early prediction of mortality risk among patients with severe COVID-19, using machine learning. Int J Epidemiol 2021 Jan 23;49(6):1918-1929 [FREE Full text] [doi: 10.1093/ije/dyaa171] [Medline: 32997743]

37. Yadaw AS, Li Y, Bose S, Iyengar R, Bunyavanich S, Pandey G. Clinical features of COVID-19 mortality: development and validation of a clinical prediction model. The Lancet Digital Health 2020 Oct;2(10):e516-e525. [doi: 10.1016/s2589-7500(20)30217-x]

\author{
Abbreviations \\ ANOVA: analysis of variance \\ DGS: Direcção-Geral da Saúde \\ DT: decision tree \\ ICU: intensive care unit \\ KNN: k-nearest neighbors \\ LGBM: Light Gradient Boosting Machine \\ MLP: multilayer perceptron \\ SMOTE: Synthetic Minority Oversampling Technique \\ XGB: XGBoost
}

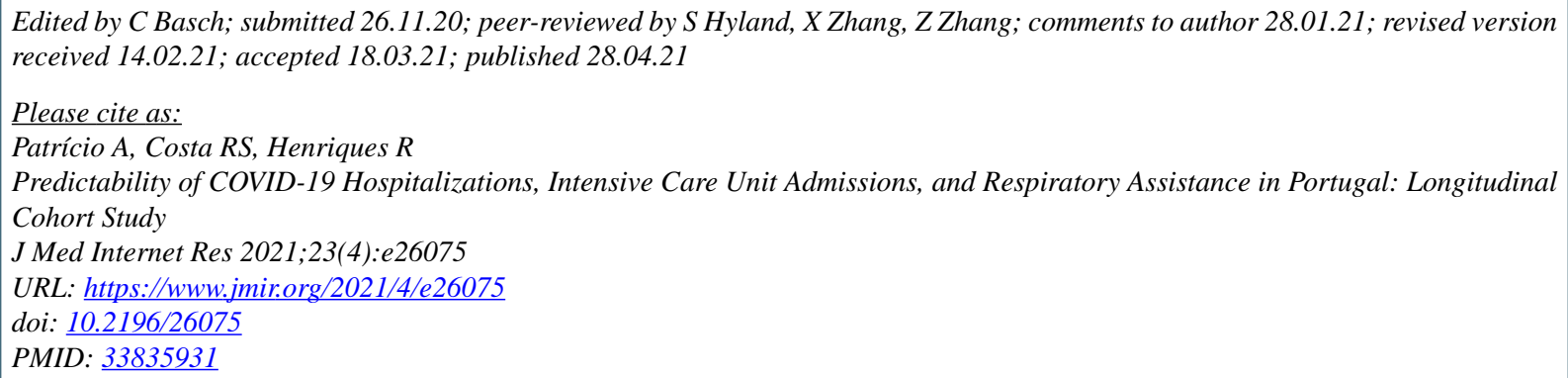

(C)André Patrício, Rafael S Costa, Rui Henriques. Originally published in the Journal of Medical Internet Research (https://www.jmir.org), 28.04.2021. This is an open-access article distributed under the terms of the Creative Commons Attribution License (https://creativecommons.org/licenses/by/4.0/), which permits unrestricted use, distribution, and reproduction in any medium, provided the original work, first published in the Journal of Medical Internet Research, is properly cited. The complete bibliographic information, a link to the original publication on https://www.jmir.org/, as well as this copyright and license information must be included. 